Historic, Archive Document

Do not assume content reflects current scientific knowledge, policies, or practices. 


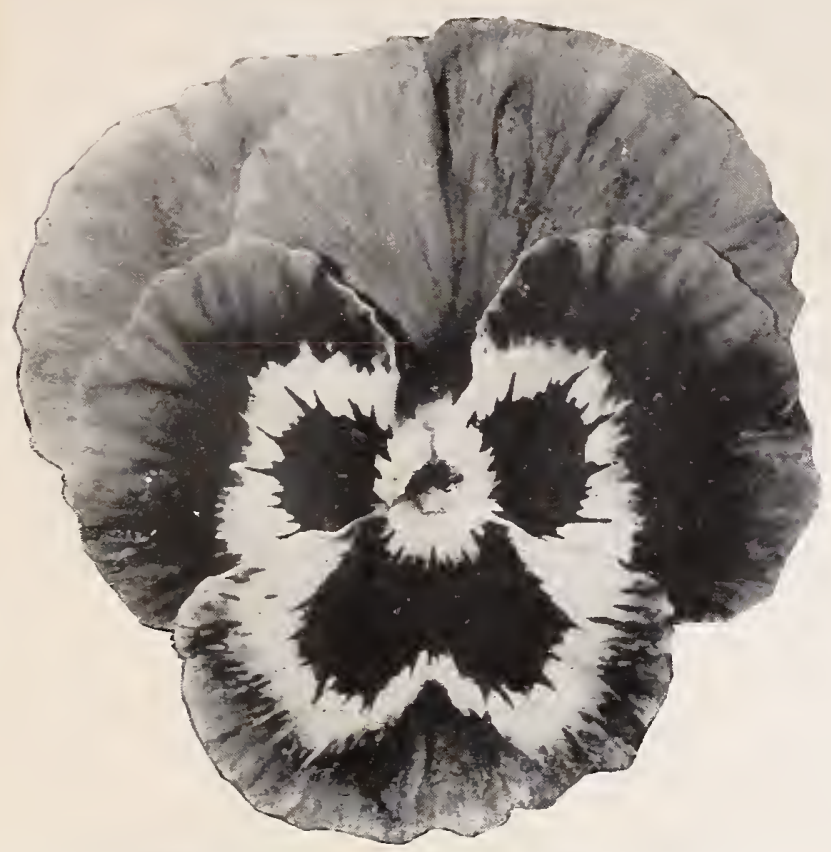

Type of bloom found in 2632-New York Giant Market Blend.
1

9

0

6

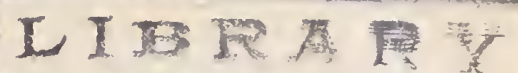

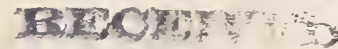

+ Jut 201923

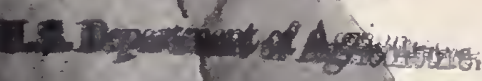

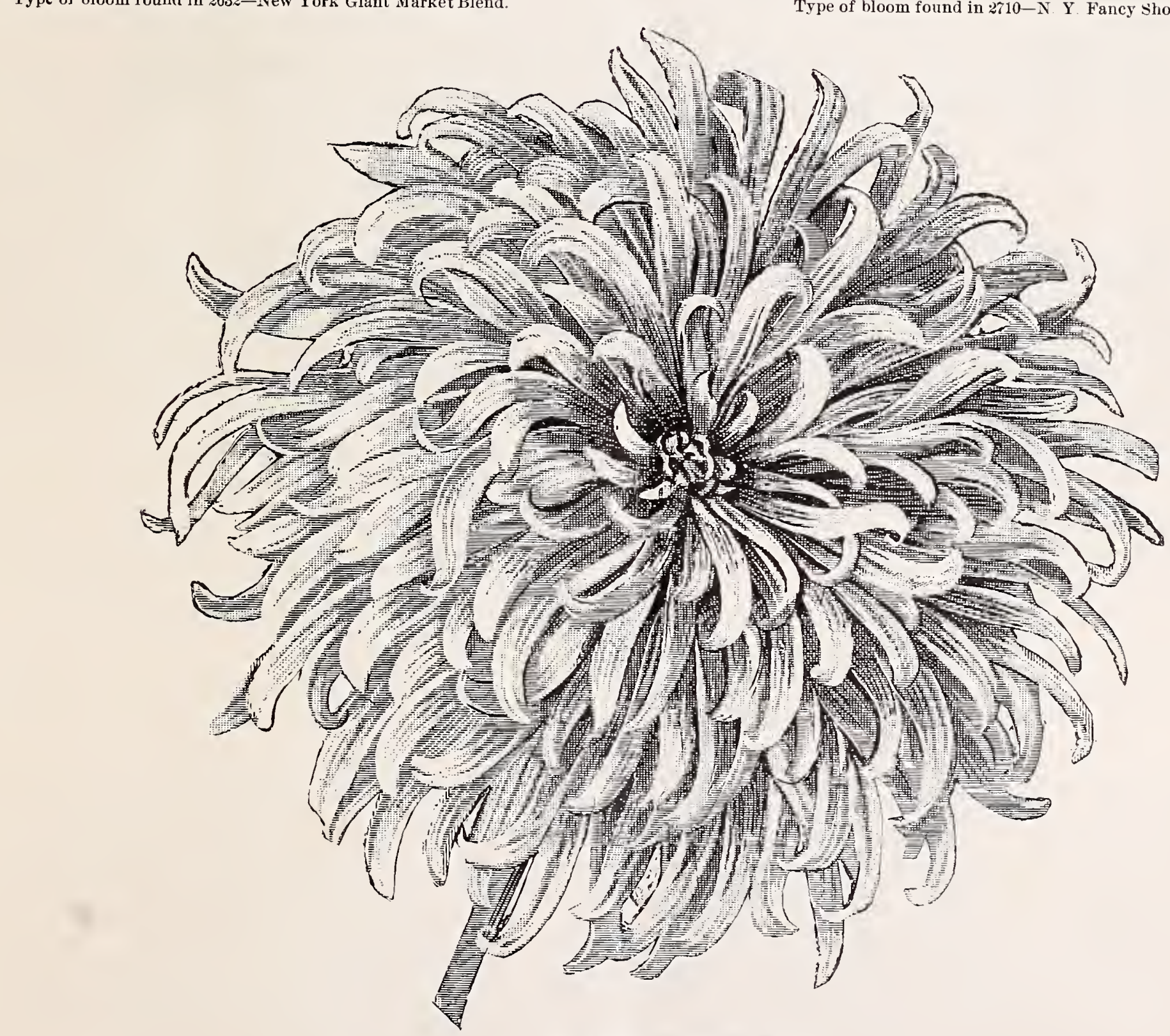

Type of bloom found in $2710-\mathrm{N}$. Y. Fancy show Blend.

1600-Aster, Giant Comet, White.

\section{Wholesale Price List of Flower and Vegetable Seeds} New York Market Gardeners' Association

P. 0. Box 1423, Manhattan Boro., New York 


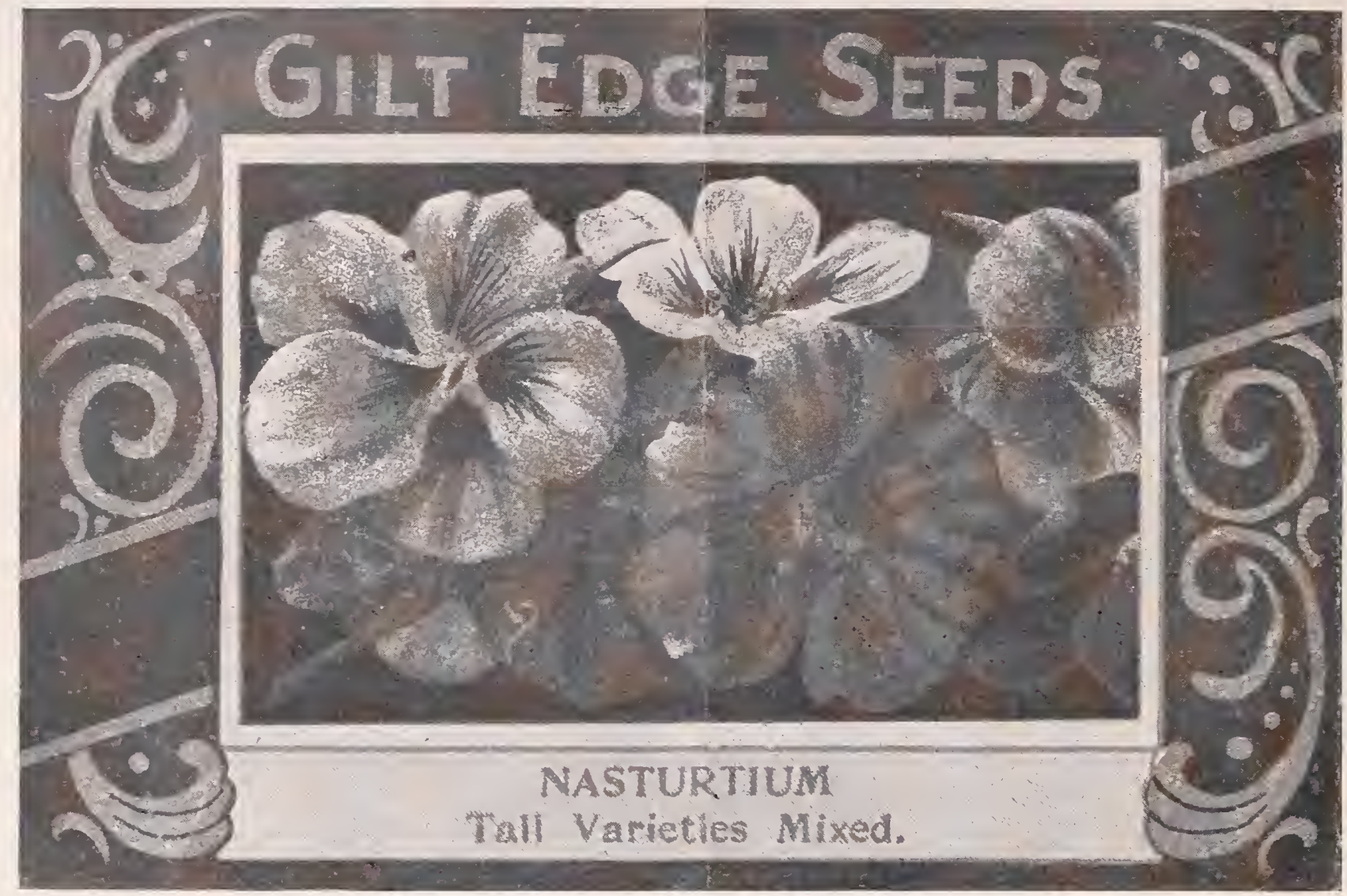

\section{Special Prices to Merchants \\ of}

Seeds in Lithographed Packets and Packages.

We offer a fine line of regetable and flower seeds in handsome colored lithographed packages and packets. These contain our best seeds and are so attractive that they sell themselves; if ordered in quantities based upon actual requirements so that none are carried over, a fine increasing trade will be the result. Order blanks naming varieties free on request.

100 pkts. $\quad 1000$ pkts.

Flower Seeds, assorted kinds in packets $\$ 065 \quad \$ 475$

Vegetable " " " " " " $" 07070750$

Vegetable " " "oz. \& $\frac{1}{4}$ pt.pkgs. $400 \quad 3750$
Sweet Peas in ounce packages.... . . _. $\$ 2 ~ \% 5 \quad \$ 2600$

Nasturtium, Tall sorts mixed ........ . $375 \quad 3500$

Nasturtium, Tom 'Thumb“ _. _. _... _ $425 \quad 4000$

In lots of 2000 or more, dealer's name and address will be printed on flower seed packets without extra cost.

Lawn Seed, Central Park Mixture, pint boses 3 \%5 3500

Lawn Seed, “ " “ $\quad$ quart " 6250000

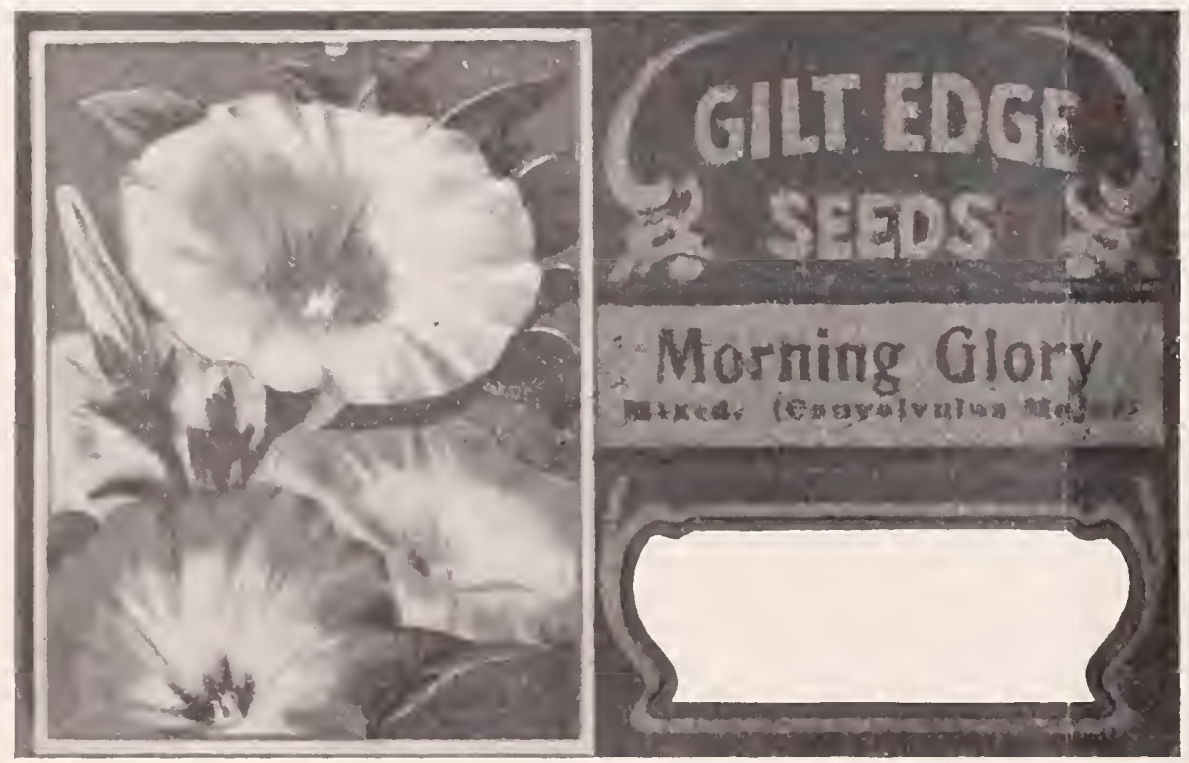

TERMS-Special Cash Discount of five per cent. (5\%) aliowed on all orders amounting to $\$ 2.00$ or more which are accompanied with a remittance. We make this offer to save bookkeeping.

Remittances should be made by Postal or Express Money Order, or by draft on New York. Local out-of-town checks cannot be accepted in small amounts unless 10c. is added for cost of collection.

Address all communications to

NEW YORK MARKET GARDENERS' ASS'N

P. 0. Box 1423

New York City 


\section{SPECIAL PRICE LIST}

\section{- \\ RICHMOND SEEDS THE FINEST THAT MONEY CAN PRODUCE.}

"THE BEST IN THE LAND."

\section{THERE ARE THREE (3) QUALITIES OF SEED.}

FIRST. Seeds that grow and mature products true to name are the best.

SECOND. Seeds of deferred growth, $i$. e., that will not germinate, becanse they are too old or have been spoiled by improper care. This can be detected by testing.

THIR Seeds that germinate or grow good, but fail to prove true to name. This class causes more damage and 1HID. disappointment than those that refuse to grow, as it takes a whole season to make the discovery.

CIITIRN Failure may result from the best or first quality seed by reason of negleet or sometimes from causes and UAU11. conditions beyond human control. 'This we cannot insure against. However, good and profitable crops cannot be produced from third or poor quality seed.

\section{FLOWER SEEDS.}

OUR OWN MIXTURES. We make up from separate colors in proper proportions, and, therefore, they are vastly superior to the mixtures as supplied by growers who plant and grow in mixture, causing the free seeding kinds to predominate. Often mixtures are the dumping places for unsalable surpluses.

PERENNIALS, Varieties of flower seeds preceded by * are hardy perennials, which have become so popular of late and are easily grown from seed.

CATALOGUE NUMBERS. For the convenience of our customers we have prefixed a number to each rariety of flower seeds, which can be used when ordering or referring to any kind.

POSTAGE. Seeds in packets, ounces and quarter-pounds we prepay to any Post Office. When ordered by the 1b. add 8c. per lb. to cover postage.

\begin{tabular}{|c|c|c|c|c|}
\hline 1478 & Abronia Umbellata, rosy lilac ............. & $\begin{array}{l}\mathrm{Oz} \\
20\end{array}$ & $\begin{array}{c}\text { Tr. Pkt. } \\
10\end{array}$ & $\begin{array}{r}\text { Pkt. } \\
3\end{array}$ \\
\hline 1480 & Abrus Precatorius (Prayer Bean) & 15 & 10 & \\
\hline 1482 & Abutilon, choice mixed & 250 & 25 & \\
\hline 1483 & Abutilon, New hybrids mixed $\ldots \ldots \ldots$ & 450 & 40 & \\
\hline 1485 & Acacia mixed, 1 b., $\$ 1.25 \ldots$ & 15 & 5 & \\
\hline $1487 *$ & Acanthus Latifolius, decorative foliage .... & 15 & 5 & \\
\hline & Achillea Ptarmica, fl. pl., Pearl ............ & 400 & 25 & \\
\hline $91 *$ & Aconitum Napellus (Monkshood) & 40 & 15 & \\
\hline $493^{*}$ & Adlumia Cirrhosa...... & 45 & 15 & \\
\hline & Adonis Aestivalis, crimson & 10 & 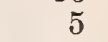 & \\
\hline $6 *$ & Adonis Vernalis.............. & 30 & 10 & \\
\hline & 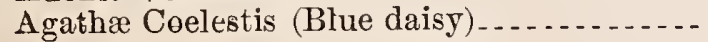 & & 20 & \\
\hline
\end{tabular}

\section{AGERATUM (Pussy Paw).}

1500 Mexicanum, blue, $1 \mathrm{~b}, \$ 1,25$

1502 Mexicanum, white

1504 Mexicanum, mixed, lb., \$1.25

1505 Mexicanum, Imperial dwarf, dark blue.....

1506 Mexicanum, Imperial dwarf, white.........

1508 Agrostemma Coeli-rosa (Rose of Heaven) ...

1509* Agrostemma Coronaria(Rose Campion) mixed

1510* A rrostemma Flos Jovis ................

Allegheny Vine, see Adlumia, No. 1493.

Aloysia Citriodora, see Lemon Verbena, No. 2356 .

Althæa Rosea, see Hollyhocks, No. 2280-96.

$\begin{array}{rrr}\text { oz. } & \text { Tr.pkt. } & \text { Pkt. } \\ 10 & 5 & 3 \\ 10 & 5 & 3 \\ 10 & 5 & 3 \\ 25 & 10 & 3 \\ 25 & 10 & 3 \\ & & \\ 10 & 5 & 3 \\ 10 & 5 & 3 \\ 10 & 5 & 3\end{array}$




\section{ALYSSUM.}

I512 Bentlramii (Sweet Alyssum), 1b., \$1.10

1511 Compactum, Little Gem.

1515 Procumbens, "Carpet of Snow" "

1517* Saxatile, "Gold dust," yellow ............

1518* Saxatile Compactum, very dwarf yellow ...

\section{AMARANTHUS.}

1520 Salicifolius (Fountain Plant)

1521 Tricolor (Joseph's Coat)...

1522 Melancholicus Ruber, blood-red

1523 Cruentus (Prince's Featlier)

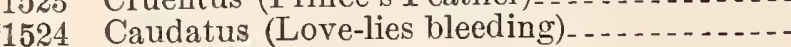

1525

Bestmixed

Ammobium, see Everlastings, No. 2104

$1527 *$ Ampelopsis Quinquefolia (Woodbine) -

1528* Ampelopsis Veitchii (Boston or Japan Iry),

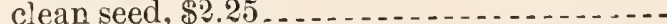

1530 Anagallis Grandifora, mixed...........

1532 Anchusa Italica, light blue

1538* Anchusa Sempervirens (Caryolopha)........

1535* Anemone (Wind Flower) Coronaria, single, from Caen; a very fine large tlowered strain 1536* Anemone Japonica, white, sweet scented ... 1538 Angelonia Grandiflora, blue, sweet scented.. 1540* Anthemis Kelwayi

\section{ANTIRRHINUM (Snapdragon).}

1543 Giant Flowering White. 1544, Firefly; 1545, Rose; 1546 , Striped; 1547 , Yellow; each.. 1548 Giant Flowering, mixed ....................

1550 Majus Niobe, crimson and white ..........

1551 Majus, tall mixed .........................

1552 Tom Thumb, large flowering mixed........

\section{AQUILEGIA (Columbine).}

\$555* Chrssantha, lg. flg. golden jellow

$1556 *$ Coerulea, lg. flg., blue and white..

155\%* Coerulea Hybrida (haylodgensis), beautiful lg. flg., long spurred variety .................

$1558^{*}$ Vulgaris, single mixed.

1559* Vulgaris, double white

$1560 *$ Vulgaris, double mixed

$1562 *$ Arabis Alpina, white

1564 Aralia Sieboldii (seeds ready in April)

1566 Arctotis Grandis (South African white daisy)

$: 568$ Ardisia Crenulata, greenhouse ornamenta plant, bearing scarlet berries; 100 seeds $60 \mathrm{c}$

1570 Arcemone Grandiflora, mixed .............

15r? Aristolochia Sipho (Dutchman's Pipe)......

1574* Armeria Maritima (Sea Pink) ..............

1576 Arnebia Cornuta (Arabian Primrose) . . . . . .

1578* Artemesia Argentea, white leaved ..........

$1580^{*}$ Asclepias tuberosa _...

1582 Asparagus Plumosus $\$ 4.00 ; 100$ seeds, 50c. grown; 1,000 seeds, $\$ 1.00 ; 100$ seeds, $50 \mathrm{c}$.

Asparagus Plumosus Robustus; 1,000 seeds,
$\$ 9.00 ; 100$ seeds, $\$ 1.00 \ldots \ldots$.
1586 Asparagus Sprengeri ; 1,000 seeds, $60 \mathrm{c} . \ldots .$.

1586 Asparagus Sprengeri ; 1,000 seeds, 60c.....

1590* Asperula Odorata Waldmeister (Maitrank).

\section{ASTERS.}

Our Aster seeds are grown by the best German specialists, excepting the American strains, and therefore should not be confounded with cheap French or Southern grown seed.

\section{CLOVENA HRANCHING。}

2591 Crimson. 1592, Lavender; 1593, Light blue; 1594, Purple; 1595, Shell-pink; 1596, White each, 1). \$5.00

1597

Our own mixture of above separate colors,

lb. $\$ 5.00 \ldots \ldots$

Mixed as imported, $1 \mathrm{~b} ., \$ 1.00 \ldots \ldots$
Oz. Tr.Pkt. Pkt.

$\begin{array}{rrrr} & 80 & 15 & 3 \\ 100 & 20 & 3 \\ 1 & & & \\ 1 & 20 & 20 & 3 \\ 15 & 5 & 3 \\ 30 & 10 & 3 \\ 15 & 5 & 3\end{array}$

0

$\begin{array}{ll}0 & 3 \\ 0 & -2\end{array}$

5

16

1630 Snow-white. 1631, Rose; 1632, Silvery lilac; each

\section{GIANT TAY OR NEEDLE.}

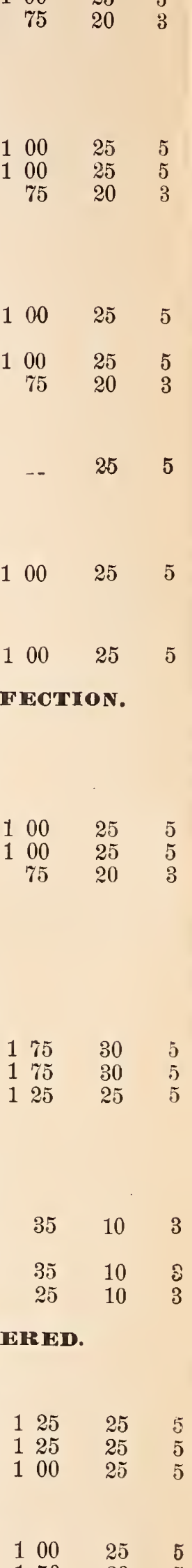

1634 White. 1635, Rose; 1636, Dark blue; eaclı_ $100 \quad 25$

TRU FIF A T'S PAEONY-FLOWERED PERTECTION.

1638 Bright crimson. 1639, Brilliant rose; 1640 , Delicate rose; 1641, Light blue: 1642, Dark blue; 1643, Lilac; 1644, Scarlet; 1645, Scarlet and white; 1646, Snow-white; 164\%, Violet and white; each . . . .............. 1648 Our own mixture of above separate colors.-.

\section{VICTORIA.}

1651 Snow-white. 1652, Azure blue; 1653, Carmine rose; 1654 , Crimson; 1655 , Crimson wlth white; 1656 , Light blue; 1657 , Dark blue; 165̋, Peach blossom; 1659, Red lilac; 1660, Rose with white; 1661, scarlet; each,

1662 Our own mixture of above separate colors... 1663

Mixed as imported.

\section{QUERN OF THE MIARIET.}

1665 Snow-white. 1666, Crimson; 1667, Light blue; 1668 , Dark blue; 1669 , Reddish lilac; 1670 , Rose; 1671 , Scarlet; each, $1 b ., \$ 4.00 .$. 1672 Our own mixture of above separate colors, $1 \mathrm{~b}, \$ 4.00 \ldots$ 1673 Mixed as imported, $1 \mathrm{~b} ., \$ 3.00$

DWARF CHR YSA NTHEMUR-FLO WERED.

1675

Snow-white. 1676 , Brilliant rose; $167 \%$, Crimson; 1678, Light blue; 1679, Dark blue; 1680, Fiery scarlet; each................ Our own mixture of above separate colors 1682 Mixed as imported ......................

\section{MISCELIANEOUS A STERS。}

1684 Ball of Fire, the brightest scarlet......... 1686 Snowball, white. .........................

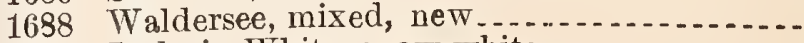

1690 Lady in White, snow-white $\ldots \ldots-\ldots . . . .$.

1692 Triumph Aster, dwarf scarlet .............

1694 Globe flowered mixed, 1b., $\$ 2.50 \ldots \ldots$

1696 German Quilled mixed, lb., $\$ 2.50 \ldots \ldots$.

1700 Single China Asters, many brilliant colors .

1701* Perennial, choice mixed . . . . . . . . . . . . .

1702* Perennial Hybrida, Grandiflora (new large flowering) .............................

$100 \quad 25 \quad 5$

Athanasia Annua, see Everlastings, No. 2106.

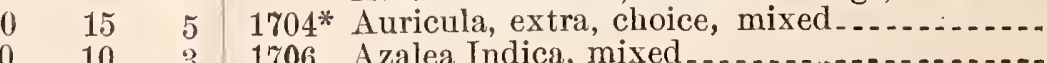
$\begin{array}{ll}4 & 00 \\ 3 & 25\end{array}$ 
Bachelor's Button, see Centaurea, No. 1881.

1708

Balsam Apple, see Gourds, No, 2208.

Balsam Pear, see Gouruls, No. 2209.

\section{BALSAM DOUBLE (Lady Slipper).}

To pick off first buds causes extra double flowers thereafter.

1709 Snow-white Perfection, best white........

1710 Prince Bismarck, bright salmon rose ........

1711 Blood-red. 1712, Fiery scarlet; 1713, Lavender : 1714 , Solferino, striped; 1715 , Camellia-flowered, mixed, each ................

1716 Fine mixed

$\begin{array}{lll}80 & 20 & 5 \\ 80 & 20 & 5 \\ & & \\ 40 & 15 & 3 \\ 20 & 10 & 3\end{array}$

$\begin{array}{rrr}20 & 10 & 3 \\ 10 & 5 & 3\end{array}$

1718* Baptisia Australis, false indigo blue .... Beartonia Aurea, see Yucca, No. 3304.

\section{BEGONIA.}

1723 Semperflorens, Vernon ....................

1724 Semperflorens, Vernon grandiflora ........

1725 Semperflorens, gracilis, brilliant rose ......

1726 Semperflorens, white $\ldots . . . . . . . . . . . .$.

1727 Semperflorens, rose $\ldots \ldots \ldots \ldots$

1728 Semperflorens, mixed 1730 Semperflorens, double flowering hybrids,

1730 Semperflorens, double flowering hybrids,

1732 Tuberous-rooted, single, mixed -..........

1738 Tuberous-rooted, crispa, frilled edges......

Rex, ornamental leaved mixed, 1,600 s., $75 \mathrm{c}$

250

$--$

150

\section{).}

\section{BELLIS PERENNIS (Double Daisy).}

1738

1739

1740

174

1742

1743

1745
1746

1748

Snowball, true, $1 / 8$ oz., $40 \mathrm{c}$

Longfellow, rose, $1 / 8 \mathrm{cz}, 30 \mathrm{c}$

Double white

Giant Maxima, mammoth white..........

Giant Maxima, mammoth pink........

Giant Ilaxima, mammoth mixed ..........

Double mixed, best German ...............

Good double mixed

Blanket Flower, see Gaillardia. Nos. 2154-64.

Bocconia Japonica ....................

Brachycome Iberidifolia, mixed ...........

Browallia Elata Grandiflora $\ldots$. Bryonopsis, see Gourds, No. 2199.

Cacalia (Tassel Flower) Coccinea . . . . . . . ...

Cactus, mixed ......................

Caladium, fancy leaved, mixed, 100 seeds, 50c
Calandrinia Grandiflor a _.................

$\begin{array}{ll}2 & 50 \\ 2 & 00\end{array}$

150

300

300

275

175

100

25

25

25

25

25

25

40

40
40

40

40

20

20

20

25

25

20

15

$\begin{array}{rrr}25 & 10 & 3 \\ 40 & 15 & 3 \\ 40 & 15 & 3 \\ -- & 15 & 5 \\ 15 & 5 & 3 \\ 150 & 25 & 5 \\ -- & 20 & 5 \\ 25 & 10 & 3\end{array}$

\section{CALCEOLARIA HYBRIDA}

1763 Fine mixed, $\frac{1}{32}$ oz., $\$ 1.25$...............

1764 Grandiflora, best lg. flg., selves mixed......

1765 Grandiflora tigered and spotted, lg. flg., mixed (James' Strain)

1767 Grandiflora dwarf, tigered and spotted .....

1768 Rugosa, shrubby, for bedding .............

1771 Calceolaria Pinnata, sulphur yellow ........

CALENDULA (Double Pot Marigold).

$17 \%$

Orange King, deep orange.............. Meteor, striped, 1b., 50c ................ Prince of Orange, dark yellow. striped, $1 \mathrm{~b}, 50 \mathrm{c}$ Favorite, sulphur yellow, striped white..... All varieties, mixed ...................... Pluvialis (Cape Marigold), single, white..... Pluvialis Pongei, double, white
California Poppy, see Eschscholtzia, Nos. 2084-88.

\section{CAIIIOPSIS.}

1784 Tinctoria, yellow, dark centre..............

1785 Nigra Speciosa, dark crimson ............

1786 Coronata Maxima, lg. flg.. yellow . . . . . . . .

1787 Drummondii (Golden Wave) ..................

1788 Hybrida Superba, new

1790 All kinds, mixed.
1794 Merlium, single Canterbury Bells, mixed ... 1795 Medium, double Canterbury Bells, mixed. 1797 Carpatica, blue, lb., $\$ 1.60$

1798 Carpatica Alba, white

1800 Macrantha, large flowering blue

1802 Calycanthema, mixed (Cup and Saucer) .....

1804 Garganica, fine for hanging baskets, \&c. 1,000 seeds, $25 \mathrm{c}$

1805 Persicifolia Grandiflora Alba............... 1806 Persicifolia Grandiflora Coerulea .......... 1808 Perennial varieties, mixed

\section{CANDYTUFT.}

1812 White Rocket, lb, 40c

1813 White, sweet scented, $1 \mathrm{~b}, 30 \mathrm{c}$

1814 Dark crimson (Dunnett's) .....................

1815 Empress, white, lb., $\$ 1.25$

1816 Little Prince, dwarf with extra large white

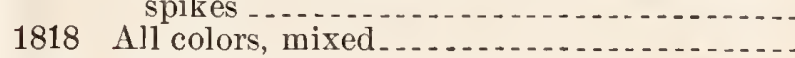

1320* Gibraltarica, lilac, shading white.

$1822 *$ Tenoreana...

1823* Sempervirens, white

$\begin{array}{rrr}10 & 5 & 3 \\ 10 & 5 & 3 \\ 10 & 5 & 3 \\ 10 & 5 & 3 \\ & & \\ 25 & 10 & 3 \\ 10 & 5 & 3 \\ 00 & 20 & 5 \\ 15 & 5 & 3 \\ 40 & 10 & 3\end{array}$

\section{CANNA.}

1825 Fine tall mixed, old type ............. 10

1826 Crozy's red-leaved varieties, mixed........

1827 Crozy's Dwarf, large flowering, mixed ......

1828 Crozy's Queen Charlotte.

Canterbury Beıls, see Campanula, Nos. 179495

Cardiospermum Halicacabum, see Balloon Vine No. 1708.

Cape Marigold, see Calendula, No. 1781.

\section{CARNATION-DOUBLE}

1831 Marguerite, white. 1832, Crimson; 1833, Rose; 1834, Scarlet; 1835, Striped; each . .........
1836

1838

1840

1841

1842

1844

1815

1846

1848

25

50

100

50

100

$\overline{2} \overline{5}$

30

10

10

10

10

10

10

10

10

10

186

1850

1852

1853

1856

5
5
5
5
5
5
5
5

\section{3}

1864

1866

1868

1870

\section{9* Cassia Marslandica}

Castor Oil Bean, see Ricinus, Nos. $3040-47$.

, sellow ground

Iarguerite, giant flowering white

arguerite, riant flowering (Malmaison) mixed .

Marguerite, dwarf mixed

Perpetual Chabaud, early flowering mixed Perpetual Mad. B. Chabaud, yellow, shading salmon, tinted cherry red, large flowering.

Perpetual Marie Chabaud, yellow, large Howering, fragrant flowering mixed renadin, fiery scarlet, early

Vienna, dwarf, early-flowered, pure white

Vienna, dwarf, early-flowered, mixed . .....

Catc

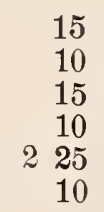

1871

1873

1874

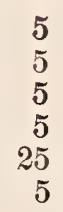

\section{3
3
3
5
3}

1878

1879

1881

1882

1884

1886

1888

\section{CELOSIA (Cockscomb).}

Cristata, Glasgow Prize, dwarf $\ldots$

Cristata, Tall mixed

Cristata, Dwarf mixed

Plumosa, Ostrich-feathered, mixed ........

Plumosa, Thompsonii Magnifica.............

Plumosa, mixed feathered ...............

Japan, scarlet

CENTAUREA.

40
80

300

$20 \quad 3$

10

250

200 
1890 Centrosema Grandiflora, climber.

Oz. Tr.Pl. Pkt.

1892* Cerastium Tomentosum,

1894 Chelone Chinese Primrose, see Primula, No. 2990.

\section{CHRYSANTHEMUM.}

1896 Coronarium, double white

1897

1898

1899

1903

1904

1905

1906

1908

1910

1912

1913

1916

1918

Tricolor Eclipse, yellow with scarlet ring

Tricolor Burridgeanum, whitc and rose....

Tricolor, Double hybrids, mixed.

Segetum Gloria, "Morning Star" "........

Segetum Helios, "Evening Star" ..........

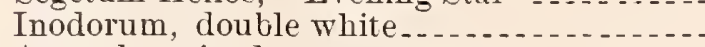

Annuals, mixed

Frutescens (Thite Paris Daisy)
Frutescens, Comtesse de Chambord (5ellow Paris Daisy)

Japonicum, early-flowering double Japanese

Japonicum, double ostrich-feathered, $1 / 8 \mathrm{oz}$, $\$ 1.00$

Cigar Plant, see Cuphea, No. 1990.

\section{CINERARIA HYBRIDA.}

1920

1921

1922

1923

1924

1920

1926

$192 i$

1928

1929

1930

1931

10

10

20

10

60

15

400

40

15

40

60

Good quality, mixed................. 450

Extra fine mixed, $\frac{1}{16} \mathrm{oz} ., \$ 1.25 \ldots \ldots \ldots \ldots$

Grandiflora, white.

Grandiftora, crimson ............................

Grandiflora, large fiowering mixed (James' strain), $\frac{1}{16}$ oz.. $\$ 2.00$ sisved from prize stock, $\frac{1}{16}$ oz. $\$ 3.00 \ldots \ldots$

Dwarf, fine German mixed.

Dwarf, large flow ering, mixed.........

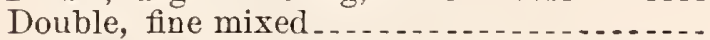

Double, dwarf, extra fine mixcd ...........

Stellata (Star Cineraria), fine for cutting ---
Radiata, a new class of Star Cincrarias, with
Coronarium, double vellow

Maximum, lg. flg., Ox-ese Daisy ..........

$\begin{array}{ll}35 & 10 \\ 25 & 10\end{array}$
long. narrow petals, turned and twisted similar to the Cactus Dahlia (see eut) ......

1935 Cineraria Maritima Candidissima, white leaved

1936 Cineraria, Diamond, new, broad white leaves

1937 Cineraria Acanthifolia, white leaved ........

1940 Clarkia, double mixed

1942 Clarkia, single mixed..

1944* Clematis Paniculata, white

1946* Clematis, large flowering, Jackman's hybrids

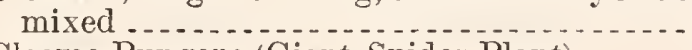

1948 Cleome Pungens (Giant Spider Plant)......

1950 Clianthus Damipieri.

1952 Cobøa Scandens, purple

1954 Cobœa Scandens, white..

Coccinea Indica, see Gourd, No. $2: 01$.

Cockscomb, see Celosia, Nes. 1863-68.

Coix Lachrymae, see Grasses, Nos. 2222.

1956 Coleus, finest mixed 1958 Coleus, fringed hybrids, extra, $\frac{1}{16} \mathrm{oz} ., 75 \mathrm{c}_{\ldots}$

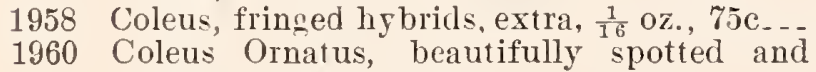
marbled, large leaves . ... . . . . . . . . . . .

Coliseum Iry, see Linaria, No. 2360.

1962 Collinsia, mixed

Columbine, see Aquilegia, Nos. 1555-60.

1964 Commelina Coelestis .... . . . . . . . . . . .

1965 Commelina Sellowiana

Convallaria, see Lily of the Valley, No. 2358.

\section{CONVOLVULUS (Morning G]ory).}

\section{0}

10

Major (Morning Glory), mixed, lb., 20c...

Major, double flowering Morning Glory .....

Mauritanicus, for hanging baskets........

Aureus Superbus, for vases . . . . . . . . . . . . . .
2022

COSMOS.

1978 Large Flowering, mixed .................

1979 Nammoth Perfection, white. 1980, Pink 1981, Crimson; each mixed

1983 Early-flowering Dawn, white, flushed pink.

1984 Giant Early-flowering, mixed ...............

1986* Corslip, mixed

1987 Crepis (Hawk weed), mixed

1988 Crrptomeria Japonica (Japan Cedar) _. . . . . . .

1989 Cryptomeria Japonica Araucarioides, fine for pots

1990 Cuphea Platycentra (Cigar Plant)

\section{CYCLAMEN PERSICUM.}

1991 Persicum, type....................... 200

1992 Persicum Album, white.......

1994 Persicum, fine mixed

1995 Persicum, double flowering mixed..........

1996 Fimbriatum, large flowering fringed varieties

1997 Papilio, Butterfly Cyclamen, mixed ..........

1998 Grandiflorum Magnificum, white spotted....

1999 Grandiflorum, Mount Blanc, pure white ....

2000 Grandiflorum Superbum. white, red eye ....

2001 Grandiflorum, Purple King (atropurpureum)

2002 Grandiflorum, Emperor William, brinht red.

2002a Grandiflorum Salmoneum, new, magniflcent bright salmon.

2003 Grandiflorum Roseum, bright rose

2004 Grandiflorum, Sweet scented ..............

2005 Grandiflorum Rokoko, large-flowered, new class with flat round flowers, petals elegantly curled and undulated.............

2005g Grandiflorum, Fine mixed as imported.....-.

2006 Grandiflorum, New York, Special Florist's blend. Our own mixture of above varieties, commencing with No. 1998 500

Cyperus Alternifolius (Crmbrella Plant) $\ldots . . .-$
Cypress Vine, white. 2011, Scarlct; 2012, Iry-leaved, each

Cypress Standing, see Ipomopsis, No. 2333.

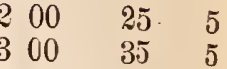

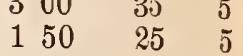

1,000 s. $100 \mathrm{~s}$. Pkt.

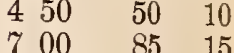

$\begin{array}{llll}7 & 00 & 85 & 15\end{array}$

$\begin{array}{lll}450 & 50 & 10\end{array}$

$450 \quad 50 \quad 10$

$\begin{array}{llll}4 & 50 & 50 & 10\end{array}$

$\begin{array}{llll}4 & 50 \quad 50 & 10\end{array}$

$\begin{array}{lll}450 & 50 & 10\end{array}$

$\begin{array}{llll}6 & 00 & 75 & 15\end{array}$

$\begin{array}{llll}450 & 50 & 10\end{array}$

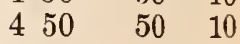

\section{DA HLIA.}

\section{SINGIE FLOWERING.}

Dahlias are as easily grown from seed as Nasturtiums and bloom freely the first season.

2018 Gracilis, striped, mixed

2020 Colossal, new, flowers measure from 8 to 9 inches across...

Twentieth Ccntury" or Orchid-forering

2024 Juarezii, Cactus-flowering ................

2026 Collerette, ncw, mixed colors

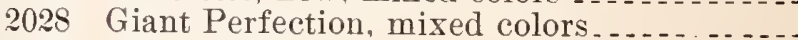

2030 Fine mixed colors . . . . . . . . . . . .

\section{DOUBLE FLOWERING.}

2032 Cactus flowered, best mixed . ............. 125

2084 .

2035 Fine double mixcd ...........................

Daisy double, see Bellis, Ncs. 1738-46.

2038* Daisy Shasta, true Burbauk stock, 1,000 seeds, 350

2040 Daisy Sit

2040 Datura Cornucopia (Horn of Plenty)

2044 Datura Wrightii, Huberiana, double mixed -

2045 Datura Humilis, Golden Qucen, enormous briiliant yellow fow

\section{"DELPHINIUM.}

2047 Cashmerianum, dark blue............... 200

2048 Chinense, large tlow ering mixed

2049 Elatum (Bee Larkspur)

2050 Formosum, blue

2052 Formosum Coelestinum

$\begin{array}{lll}10 & 25 & 5 \\ 20 & 10 & 3 \\ 50 & 15 & 3 \\ 35 & 15 & 3 \\ 50 & 20 & 3\end{array}$


2053 Nudicaule, scarlet.

2054 Hybridum, lg. flg., single mixed ........

2055 Hybridum, double mixed ....

2056 Zälil (Sulphureum), y ellow

Dianthus Barbatus, see Sweet William, Nos. 3226-34.

Dianthus Caryopligllus, see Carnations, Nos. 1831-56.

Dianthus Chinensis see Pinks. Nos. 2878-80.

2058 Didiscus Coeruleus, lavender blue.........

Digitalis, see Foxglove, Nos. 2144-46.

Dolichos, see Hyaeinth Bean, Nos. 2307-09.

2060 Dracaena Australis $\ldots \ldots 1$ Dracaena Indivisa, $16 ., \$ 2.00 \ldots \ldots$

2062 Dracaena Indivisa Lineat a, lb., \$2.50 ......

Dusty Miller, see Centaurea, No. 1879.

Dutchman's Pipe, see Aristolochia, No. 1572.

2064 Echeveria Glauca No. 3301 .

2066* Echinops Ritro

Edelweiss, see Gnaphalium, No. 2190.

English Clover, see Everlastings, Nos. 2110-16.

2068* Eremurus Robustus, giant flowering, 100 seeds, $\$ 1.00$

$2070 *$ Eremurus Turkestanicus, 100 seeds, $50 c$

2072* Eremurus Himalaicus Majestic, 190 seeds, $75 \mathrm{c}$

20 4 * Erigeron Aurantiacus Hybridus, large fiowering Hybrids of the Orange Daisy in great variety of colors.

$20 ; 6$ Erigeron Grandiflorus Elatior, new, pale lilac, early flowering.

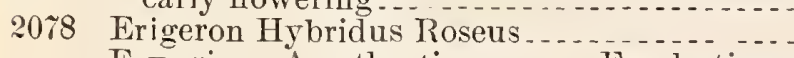

Eryngium Amethystinum, see Everlastings, No. 2108 .

2080 Erysimum Perof skianum, orange ..........

2082 Erythrina Crista Galli (Coral Plant) West, very large flowered.

2085 Eschscholtria Mandarin

2086 Eschecholtzia Rose-cardina], lg. flg., rose...

2088 Eschscholtzia, mixed ...................

2090 Eucharidium Breweri, rose .... . . . . . . . . . .

Eulalia Japonica Zebrina, see Grasses, No. 2239.

2092* $\begin{aligned} & \text { Eupatorium Fraseri, white } \\ & 2094 \\ & \text { Euphorbia Variegata, variegated foliage... }\end{aligned}$

2095 Euphorbia Variegata, Variegated foliage...-

2097 Euryangium sumbul (Perennial MIusk Root),

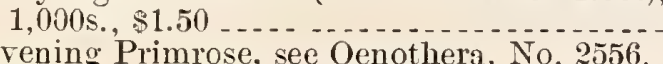

EVERLASTINGS (Straw Flowers).

2100 Acroclinium, single mixed...............

2101 Acroclinium, double mixed

2104 Ammobium Grandiflora, lg. dbi., white.....

2106 Athanasia Annua, yellow .............

2108 Eryngium Amethystinum

2110 Globe Amaranth(Gomphrena) Aurea Superba

2114 Globe Amaranth, Nana Compacta, dwarf red

2116 Globe Amaranth, mixed .......

2118 Helichrysum IIonstrosum, double mixed --

2120 Helipterum Sanfordii, yellow ............

2121 Helipterum Corymbifiorum, white $\ldots . . . . .$.

2123 Rhodanthe Maculata, mixed $\ldots$

2124 Xeranthemum, double mixed

2126 Fenzlia Dianthiflora _... . . . . . . . . . 250

Ferns, Greenhouse, mixed.

Feverfew, see Matricaria, No 2420

Flora's Paint Brush, see Caealia, No. 1756

Flowering Flax, see Linum, Nos. 2364-66.

Flowering Maple, see Abutilon, No. 148\%.

Flowering Sage, see Salvia, No. 307r.

FORGET-ME-NOT (Myosotis),

2130 Alpestris, blue ..........................

2131 Alpestris Robusta Grandiflora

2132 Alpestris Stricta Grandiflora, Royal blue....

2133 Alpestris Victoria, dwarf, blue, best for pot

culture Dissitiflora, early, deep blue......

2136
$60 \quad 15 \quad 3$

$\begin{array}{lll}50 & 15 & 3 \\ 20 & 10 & 3\end{array}$

$25-10-3$

$100 \quad 25 \quad 5$

$20 \quad 10 \quad 3$

\section{GAILIARDIA (Blanket Flower)}

2154 Amblyodon, blood-red

2155 Picta, red and yellow.

2156 Picta, Lorenziana, double mixed

2158 Picta, mixed, $1 \mathrm{~b} ., 75 \mathrm{c}$

Kermesina Splendens 150

- 150

$2164^{*}$ Grandiflora, newest varicties, mixed

15
10
15
10
50
150
150
50
40

$\begin{array}{rrrr}1 & \tilde{5} 0 & 25 & 10 \\ -- & 20 & 5 \\ & & & 10 \\ 1 & 00 & 20 & 5 \\ & & \\ -- & 25 & 5 \\ -- & 25 & 5 \\ & & \\ 10 & 5 & 3 \\ 50 & 20 & 5 \\ & & \\ 15 & 5 & 3 \\ 20 & 10 & 3 \\ 20 & 10 & 3 \\ 10 & 5 & 3 \\ 75 & 15 & 3\end{array}$

218

2182 Grandiflora, large flowering mixed

2183 Grandiflora, tigered aud spotted, extra .....

2184 Grandiflora, crassifolia erecta .

2185 Grandiflora, Crassifolia horizontalis .......

2186 Gigantea, immense, from 4 to 5 inches across

2192 Godetia, best mixed 1b. \$1.00 (Edelweiss) ... Gold Dust, see Alyssum, No. 1517 Golden Feather, see Pyrethrum. No. 3028. Gomphrena, see Everiasiings, Nos. 2110-16.

\section{GOURDS-ORNAMENTAL.}

2196

\section{7}

2198

2199

2200

2201

2203

2204

2205

2206

2207

2210

2211

2212

2213

2215

2217

Abobra Viridiflora

Apple-shaped

Eryonopsis Laciniosa

Calabash, or Dipper.

Coccinea Indica.....

Dish Cloth

Gooseberry

Hedgehog.

Hock Orange.

Momordica Balsamina (Balsam Apple).... . .

Momordica Cherantia (Balsam Pear)........ 15

Nest Egg

Pomegranate.

Snake Cucumber

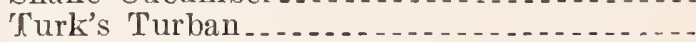

15

50

$\overline{40} \quad 15$

25

25

10

100

10

20
5

5

\section{GRASSES-ORNAMENTAL. ANNUAL SORTS.}

2220 Agrostis Nebulosa. .

$\begin{array}{rrrr} & 30 & 10 & 3 \\ & 40 & 15 & 3 \\ 1 & 50 & 20 & 5 \\ & & & \\ 1 & 75 & 15 & 3 \\ 1 & 50 & 20 & 3 \\ 2 & 50 & 30 & 5\end{array}$

2221 Briza Maxima (Quaking Grass)

2222 Coix Lacrymæ (Job's tears), lb., $40 \mathrm{c} \ldots . . . .$.

2223 Pennisetum Longistylum (villosum) ..........

2224 Pennisetum Rueppelianum (Purple Fountain Grass)

2225 Zea Gracillima Gigantea Variegata, lb., $40 \mathrm{c}$.

2226 Zea Japonica Foliis Variegatis (Striped Japanese Maize) . . . _ : = = = = . . . . . . . . . . . . 


\section{PEFENNIAL VARTETES.} eass culture. They are becoming very popular for borders, groups and massing.

2228 Andropogon Argenteus, for groups........ 2230 Arundo Donax, stately Reeds ............

2231 Arumdo Donax Foliis Variegatis, very decorative . . . . . . . . . . . . . . . . . . . . .

Bromus Brizæe formis, for bouquets, lb., $40 \mathrm{c}$. Cryptopyreum Richardsonii. ... . . . . . . . . Elymus arenarius . . . . . . . . . . . . . . . . . . . Eriantbus Ravennæ

Eulalia Japonica, for clumps _............

Eulalia Japonica Zebrina, extra fine......

Festuca Glauca. for edgings ..... . . . . . . . .

Gymnothrix Caudata, for groups.........

Gymnothrix Latifolia.

Gynerium Argcnteum (Pampas Grass) ......

Gynerium Argenteum, roseum for clumps ..

Grnerium Argenteum Vrriegatum, fine....

Panicum Sulcatum, for clumps...........

Stipa Pennata (Feather Grass) .....
Stipa Elegantissima, for bouquets
The following are all hardy perennials of

Oz. Tr.Pkt. Pkt.

2254 Grevillea Robusta (Silk Oak)

2256* Gunnera Scabra, ornamental leaved........

2258 Gypsophila Elegans, white..

2259 Gypsophila Muralis, rose

2260* Gypsoplila Paniculata, white, fine for bouquets, lb., $\$ 1.50$

Hawkweed, see Crepis, No. 1987

2964 Hedzsarum Coronarium, mixed

Helianthus, see Sunflower, Ncs. 3174-80.

Helichrysum, see Everlastings, No. 2118.

2266 Heliotrope, fragrant sorts mixed

2267 Heliotrope, Lemoine's Giant mixed....

Helipterum, see Everlastings, No. 2120-21.

2270* Helleborus (Christmas Rose) Niger . . . . . . . .

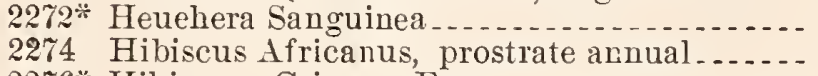

2276* Hibiscus, Crims on Eye. $227 \%$ Hibiscus, Giant Yellow or Golden Bowl

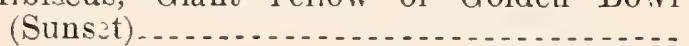

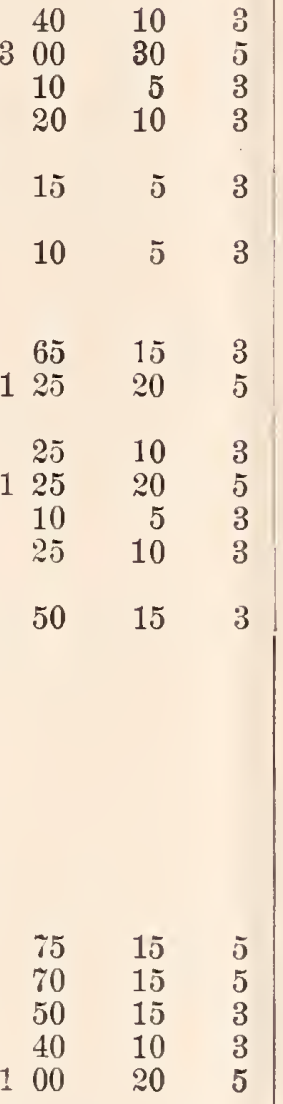

2280 Chater's Apple-blossom. 2281, Chestnut brown; 228:, Crimson; 2283, Golden rellow: 2284, Lilac; 2285, Salmon pink; 2286, Scarlet; 228\%, Snow-white; 2288, Sulphur zellow; each, lb., $\$ 10.00 \ldots . . . . . . . . . .$.

2290 Chater's prize strain, mixed, 1 b., $\$ 8.00 \ldots \ldots$

2292 Double mixed, good quality .............

2294

2296

Allegheny, fringed, double mixed. ........

Honesty, see Lunaria, No. 2388.

2300 Humea Elegans, $\frac{1}{16}$ OZ., $75 \mathrm{c} . .$.

2302 Humulus Japonica (Japan Hop)

2303 Humulus Japonica Variegata ..

2305 Hunnemannia Fumariæfolia, fine for cutting

2307 Hyacinth Bean (Dolichos Lablab), mixed, lb.,

2308 Hyacinth Bean, Daylight, lb., $60 \mathrm{c}$

2309 Hyacinth Bean, Purple Soudan, lb., $60 \mathrm{e} . . .$.

2312 Hyacinthus Candicans, 1 b., $\$ 1.00 \ldots . . . . . . .$.

Iberis, see Candytuft, Nos. 1812-23.

Ice-Plant, sec Iiesembryanthemum, No. 2430.

2816 Impatiens Sultanii, $\frac{1}{16} \mathrm{oz}, 75 \mathrm{c} \ldots \ldots . .7$.

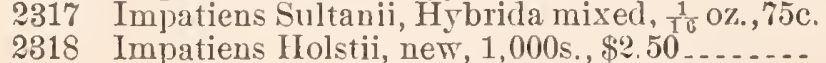
Impatiens, see Balsams, Yos. 1709-16.

Imperial IIorning Glory, see Ipomœa, No. 2329. Imperial Pink, see Pinks, No. 2881.

2320* Incarvillea Delavayi, magnificent flowers similar to Gloxinias, 100 seeds, $40 \mathrm{c} . . . . .$. India Pink, see Pinks, No. 2878.

Indian Shot, sce Canna, Nos. 1825-28.

2322 Bona Nox, blue (Evening-Glory), 1b., 75c .. 2324 Coccinea, scarlet star ..................... 2326 Moonflower, large white, black-seeled ...... 2327 Ioonflower, large white, white-seeded ...... 2328 Heavenly Blue......................... .. 2329 Imperial, Japanese Morning-Glory, mixed .2330 Leari, blue dawn flower .................. 2331 Brazilian Morning-Glory (Setosa) ........... Purpurea see Convolvulus, No. 1970. Quamoclit, see Cypress Vine, Nos. 2010-14.

2333 Ipomopsis Elegans, mixed

$2334^{*}$ Iris.Kaempferi, newest hybrids mixed..... Jacobœa, see Senecio, No. 3107.

Jacob's Ladder, see Polemonium, No. 2920.

Job's Tears, see Grasses. No. 222?.

Joseph's Coat, see Amaranthus, No. 1521.

2336 Kaulfussia, Amelloides _...

2338 Kochia Scoparia (Summer Cypress) .......

Koniga Maritima, see Alyssum, No. 1512.

Kudzu, see Puaria, No. 3026.

2340 Lantana Hrbrida, fine mixed

2342 Lapageria Rosea, greenhouse climber, 100 seeds, $\$ 1.00$

\section{LARKSPUR.}

2344 Dwarf Rocket, mixed ......................

2346 Tall Rocket, mixed ...................

2348 Candelabrum, dwarf branching, mixed.... .

2350 Emperor, mixed .........................

$\begin{array}{lrr}\text { Oz. } & \text { Tr. Pkt. } & \text { Pkt. } \\ 10 & 5 & 3 \\ 10 & 5 & 3 \\ 40 & 10 & 3 \\ 40 & 10 & 3 \\ 50 & 15 & 3 \\ 10 & 5 & 3 \\ 60 & 15 & 3 \\ 20 & 10 & 3\end{array}$

Lathyrus Latifolius, see Peas, Nos. 2792-94.

2352 Lavatera Arborea Variegata . . . . . . . .

$2353^{*}$ Lavatera Cachemiriana, new; large flowering rose color, $\frac{1}{16}$ oz., $\$ 1.50 \ldots \ldots \ldots$

2354 Lavatera Trimestris, mixed ...............

2356 Lemon Terbena $\ldots$. $235 S^{*}$ Lily of the Valley, seed, lb., $\$ 5.00 \ldots \ldots \ldots$

2360 Linaria Cymbalaria (Kenilworth Ivy) .......

2361 Linaria Cymbalaria, White-fluwered Kenil-

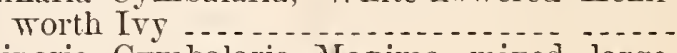

2362 Linaria Cymbalaria Maxima, mixed, large,

2364 Linum Grandiflorum Rubrum, lb., $60 \mathrm{c}_{\ldots} \ldots .$.

2365* Linum Flarum ......................

$2366^{*}$ Liuum Perennial, mixed................. 15

$\begin{array}{lll}20 & 10 & 3 \\ 00 & 25 & 5\end{array}$

Lippia Citriodora, see Lemon Verbena, No. 2356.

\section{LOBELIA.}

23r0 Erinus (Gracilis), blue . . . . . . . . . . . . . . 2371 Erinus Emperor William, light blue .......

2372 Erinus Prima Donna, crimson, white ere... 2373 Erinus Speciosa, Crystal Palace, trailing.... 2374 Erinus White Lady Compacta, new ........ 2376 Erinus Crystal Palace Compacta, true ...... 2378 Erinus Finest, mixed ...................... 2380* Cardinalis (Cardinal Flower) .............. $2381 *$ Fulgens, Queen Victoria, $\frac{1}{32}$ nz., $\$ 1.00 \ldots \ldots-$
$2382^{*}$ Fulgens, Best Perennial Hybrids, mixed, $\frac{1}{3}$ OZ., $\$ 1.00$

candens, climber 2384 Lopł 10 spermum Scandens, climber
2386 Lotus Peliorhynchus (Coral Gem), five for

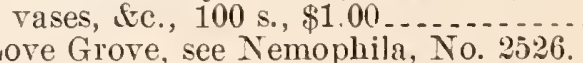

Love-in-a-Mist, see Nimella, No. 2538.

Love-Lies-Bleeding, see Amaranthus, No. 1524.

2388 Lunaria (Honesty) Bicnnis ................

2390* Lupinus, annual sorts, mixed, lb, 40c....mixed

2394* Lychnis Chalcedoniea (Ragged Robin), scarlet 2396* Lychuis Haageana Hrbrida, mixed........ 2398* I.ychnis Fulgens, fiery scarlet . . . . . . . . . . .

2400* Lythrum Roseum Superbum . ............. "Maitrank," see Asperula, No. 1590 .

2402* Malva Moschata, white, scented mallow ...

MARIGOLD.

$25 \quad 10$

2404 African, double mixed, lb., \$1.00

2405 African, El Dorado, mammoth flowers....

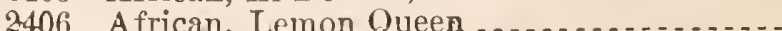

$\begin{array}{rr} & 40 \\ & 85 \\ 1 & 00 \\ & 35 \\ & 50 \\ 1 & 00 \\ 25 & 25 \\ 2 & 00 \\ & --\end{array}$

3
3
3
3

3
10
3
5
3
3


2407 African, Nugget of Gold.

2408 Afriean, "Pride of the Garden," early, dwarf

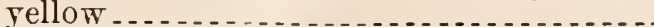

2409 African, Prince of Orange ............

2412 French, Tall double mixed

2414 French, Dwarf double mixed 1b.

2416 French, Gilt Edge, dwarf single, striped ....

2417 French, Legion of Honour, single yellow, brown spotted

Marvel of Peru, see Four O'Clock, No. 2142.

2420 * Natricaria Capensis (Double White Feverfew)

2421* Matricaria Eximia, Golden Ball, hardy perennial, suitable for beds or borders, bearing double yellow flowers

2424 Matthiola Bicornis, fragrant .............

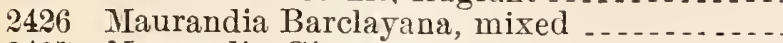

2427 Naurandia Giant blue, new 2430 Mesembryanthemum Crystallinum, Ice-Plant clean seed, lb., $\$ 1.25$

2432 Mesembryanthemum Cordifolium Variegatum, for bedding, $\frac{1}{8} \mathrm{Oz}$., $50 \mathrm{c}$

2434 Mesembryanthemum, Tricolor (Dew Plant) Mexican Poppy, see Argemone, No. 1570.

\section{MIGNONETTE.}

2436 Large Flowering Sweet, lb., 40c . . . . . . . ...

2438 Grandiflora, Golden Queen

2439 Grandiflora, Orange Queen, distinct color...

2440 Grandiflora, Defiance, lb. $\$ 2.50$

2442 Grandiflora, Gabriele, very large spikes .....

2443 Grandiflora, Giant Pyramidal, 1b., $\$ 2.50 \ldots$.

2444 Grandiflora, Goliath, red, new ............

2445 Grandiflora, Machet, for pots 2446 Granditlora, Golden Machet, $1 \mathrm{~b} ., \$ 2.50 \ldots$

2447 Grandiffora, Machet Ruby, large coppery-red flowers . .

2448 Grandiflora, Bismarck, improved dwarf

2449 Grandiflora, Miles Hybrid Spiral, true, lb., $\$ 1.25$.

2450

Mimosa Pudica, see Sensitive Plant, No. 3108.

2452

2454 Mimulus Moschatus (Musk Plant) .......

Mirabilis Jalapa, see Four O'Clock, No. 2149

Momordica, see Gourds. Nos. 2208-09.

Noon Flower, see Ipomœa, Nos. 2326-27.

Monkey Flower, see Mimulus, No. 2453.

Monk's Hood, see Aconitum, No. 1491.

Morning Glory, see Convolvulus, No. 1970.

Mountain Fringe, see Adlumia. No. 1493.

Mountain of Snow, see Euphorbia, No. 2094.

Mourning Bride, see Scabiosa, Nos. 3090-98.

2460 Musa Ensete, 1,000s., $\$ 7.00 ; 100 \mathrm{~s}$, 80c

Myosotis, see Forget-me-not, Nos. 2130-39.

\section{NASTURTIUM.}

When ordering by the $1 b$. to be sent by mail add 8 cents per $1 b$, to cover postage.

\section{DWAFF OR TOM THUMB VARIETIES.}

2464 Aurora, deep yellow with carmine blotches.-

Chamæleon, great variety of colors on one plant
Mimulus Cardinalis Grandiflorus............

2538 Nigella Damascena (Love-in-a-mist) .........
Lo. $1 / 41 \mathrm{~b} . \quad \mathrm{Oz}$

$50 \quad 20 \quad 10$

$60 \quad 20 \quad 10$

$75 \quad 20$

$60 \quad 20 \quad 10$

$\begin{array}{lll}60 & 20 & 10\end{array}$

$50 \quad 20 \quad 10$

$\begin{array}{lll}60 & 20 & 10\end{array}$

$75 \quad 20 \quad 10$

$75 \quad 20 \quad 10$

\%5 $20 \quad 10$

$60 \quad 20 \quad 10$

$60 \quad 20 \quad 10$
$75 \quad 20 \quad 10$
2526 Nemophila, fine mixed

2528 Nicotiana Affinis, white fragran

2529 Nicotiana Colossea, ornamental foliage plant,

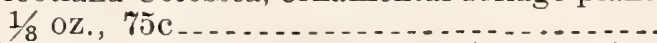

2530 Nicotiana Sanderæ, new, star-lize carmin rea flowers; a free bloomer

2531 Nicotiana Sylvestris, long, pure white, tubeshaped flowers; fiue for groups...

2534 Nierembergia Gracilis, light blue

2535 Nierembergia Frutescens, lilac

2539 Nigella Hispanica (Spanish)

2542 Nolana, mixed, lb., 50c .................

2544 Nycterinia Capensis, white, fragrant

2556 Enothera (Evening Primrose), mixed.

2560 Oxalis, rosea mixed...

2561 Oxalis Troproloides...

2564* Pæony, herbaceous, double mixed

\section{PANSY.}

We are headquarters for everything known in Pansies. Our special Pansy price list, containing 20 photo's of latest novelties and specialties, free on request.

\section{Section 1.-NOVELTIES.}

$\begin{array}{ccc}\text { Lb. } & 1 / 4 \mathrm{Lb} . & \mathrm{Oz} \\ 60 & 20 & 10 \\ 60 & 20 & 10 \\ 50 & 20 & 10 \\ 60 & 20 & 10 \\ 60 & 20 & 10 \\ 60 & 20 & 10 \\ 45 & 15 & 5 \\ 70 & 25 & 10\end{array}$

60

20

$20 \quad 10$

$20 \quad 10$

$20 \quad 10$

$20 \quad 10$

$20 \quad 10$

$20 \quad 10$

2010

$20 \quad 10$

$20 \quad 10$

$20 \quad 10$

$20 \quad 10$

2010

$20 \quad 10$

$20 \quad 10$

155

75

251

$60 \quad 20$

$\begin{array}{lll}75 & 25 & 10\end{array}$

$60 \quad 25 \quad 10$

100

10

20

15

15

$\overline{75} \quad \overline{2} \overline{5} 10$

$\begin{array}{lll}75 & 25 & 10\end{array}$

$\begin{array}{lll}50 & 45 & 15 \\ 50 & 10\end{array}$

oz. Tr. Pkt. Pkt

$\begin{array}{rrr}10 & 5 & 2 \\ 20 & 10 & 3\end{array}$

25

$400 \quad 35 \quad 10$

$\begin{array}{rrr}15 & 5 & 3 \\ 35 & 10 & 3 \\ 25 & 10 & 3 \\ 10 & 5 & 3 \\ 10 & 5 & 3 \\ 10 & 5 & 3 \\ 60 & 15 & 3 \\ 10 & 5 & 3 \\ 100 & 25 & 3 \\ 00 & 15 \\ 50 & 15 & 3 \\ 70 & 20 & 5\end{array}$

2658a Orange, new color, very fine

2688a Giant Curled Preciosa, crimson rose, white ed re with five blotches

2688b Giant Curled Psyche, broad white curled and crinkly edge giants with five violet blue blotches...........

2688 c Giant Curled Corona, citron yellow,
petals richly veined, brilliant red, with black eye

26s8d Giant Curled Hero, lower petals deep

Oz. Tr. Pkt. Tr. Pkz. Pkt. $5,000 \mathrm{~s}$. $2,0000.125 \mathrm{~s}$.

$450 \quad 180 \quad 90 \quad 11$

$450 \quad 180 \quad 90$

$1000 \quad 400 \quad 200$

25 blue, upper petals lighter color

500 
208\&e Giant Curled Cardinal, dark velvety red with large blotches............ 2688f Giant Curled Vulcano, 5-blotched on deep red ground .

$2688 \mathrm{~g}$ Giant Curled Andromeda, delicate apple-blossom ground, flushed and veined lavender, blue and rosy iilac. Small pkts. only, 25c.

2689a Giant Curled IIagnifica, broad undulated petals overlapping each other, giving the appearance of being semidouble.

2692a Improved Parisian Giants, flowers 3 to $31 / 2$ inches across, sweet scented

2692b Improved Parisian Giant White, Emperor Franz Joseph, dwarf compact habit; large violet blue blotches. Small pkts. only, 25c.

2800a Giant Cassier, Fancy Red; conspicuous for its scarlet, crimson and purplish-red colorings. and color; upper petals long, grooved and curled: lower petals yellow, blotched and streaked _........... beautiful 4-colored variety -...-violet blotches on lower petals.....

2716b Giant Rosy Morn, violet blotched, white-margined crimson purple -...

2\%1\%a Giant Mauve Qneen, upper peials delicate rosy lilac, lower petals blotched $20 \mathrm{c}$

2\%20b Giant Prince Henry, velvety deep indigo blue with golden centre blotched blackich brown ...........

2721 Giant Crown Prince, brown-black with golden bronze face _..........

2731 Giant Indigo Blue. brightest and richest blue produced in pansies

2739 Giant Diana, cream colored .........

2741 Novelty Pansy collection 1 pkt. each of above 22 kinds, regular value $\$ 3.49$, for . . . . . . . . . . $\$ 1.75$ net.

$\begin{array}{rrrrrrr}8 & 00 & 3 & 20 & 1 & 60 & 20 \\ 5 & 00 & 2 & 00 & 1 & 00 & 12 \\ 6 & 00 & 2 & 40 & 1 & & \\ 3 & 00 & 1 & 20 & & 60 & 15 \\ 8\end{array}$

$\begin{array}{lllllll}12 & 00 & 4 & 80 & 2 & 40 & 30\end{array}$

$\begin{array}{llllll}4 & 00 & 1 & 60 & 80 & 10\end{array}$

$\begin{array}{llllll}3 & 00 & 1 & 20 & 60 & 8\end{array}$

$\begin{array}{lllll}3 & 00 & 1 & 20 & 60\end{array}$
$2715 \mathrm{c}$ Giant Peacock, flowers vely large - -
$2716 \mathrm{a}$ Giant Purple King, purplisu-red with bluish carmine. Small pkts. only,

$\begin{array}{lllllll}5 & 00 & 2 & 00 & 1 & 00 & 12 \\ 6 & 00 & 2 & 10 & 1 & 20 & 15\end{array}$

$\begin{array}{llllll}4 & 50 & 1 & 80 & 90 & 11\end{array}$

$\begin{array}{llllll}4 & 00 & 1 & 60 & 80 & 10\end{array}$

$\begin{array}{lllllll}4 & 00 & 1 & 60 & 1 & 80 & 10\end{array}$

$\begin{array}{lllllll}5 & 00 & 2 & 00 & 1 & 00 & 12 \\ 2 & 00 & 4 & 80 & 2 & 40 & 30\end{array}$

2
2
2
2
2
2
2

2
2
2
2
2
2
2
2
2

2687 Peacock, upper petals peacock blue,
lower petals claret-red white margin

2699a Giant Cassier Bridesmaid apple-

2700 Cassier Giant Blotched, 3 and 5

2703 Bugnot s Superb Giant, true 2706 Giant Imperial, saved from the best

2720a Giant Prince bismarck, brown and eye, lower petals blotched purple.

$\begin{array}{llllllll}1 & 00 & 0 & 40 & 0 & 20 & 0 & 05\end{array}$

2633 Atrosanguinea, darkest blood red

2639 Purple and Gold; lower petals blotched on cream and gold, upper petals velvet purple, banded gold .

2641 Claret and Purple; extra, 5 blotched. on pink and white ground

2642a Giant Freya, large purplish-violet flowers, broadly margined white...

2643a Kamerun, velvety blackish-brown without face ground with large, dark violet blotches .... . . . . . . . . . . . . . . .

$2646 \frac{1}{2}$ Silver Seam, dark black-brown, with broad silver-white ed ge ........... .

2647 Black Prince or Knight, rich glossy.

2647a Black, Red and White edged 26 airy Queen, brilliant sky-blue, margined silvery-white

2651 Coquette de Poissy, rose-tinted, pale blue of the Cattleya .

2655 Silver-Bordered Maroon and Purple, lower petals brown, blotched on cream ground.

2656 Bronze, Scarlet and Gold Margined.

2657 Giant Golden Gem or Queen, golden yellow ; no eye.................. .

2660 Gold-Banded or Gilt-Edged, orange and red.

$2660 \frac{1}{2}$ Crimson and Gold, bright velvety, crimson gold-flaked ground, with five distinct dark blotches, narrow gold-frilled edge .

2665 a I)iana, pure cream colored

$28 \% 0 \frac{1}{2}$ Giant Snow Queen; no eye.........
$300 \quad 120 \quad 60$

$\begin{array}{lllll}4 & 00 & 1 & 60 & 80\end{array}$

$125 \quad 50 \quad 25$

$150 \quad 60 \quad 30$

$150 \quad 60 \quad 30$

$100 \quad 40 \quad 20$

$\begin{array}{llll}1 & 00 & 40 & 20\end{array}$

$150 \quad 60 \quad 30$

$\begin{array}{lll}80 & 32 & 16\end{array}$

$\begin{array}{lll}70 & 28 & 14\end{array}$

$150 \quad 60 \quad 30$

$150 \quad 60 \quad 30$

$125 \quad 50 \quad 25$

$100 \quad 40 \quad 20$

$\begin{array}{rrrr}1 & 50 & 60 & 30 \\ 1 & 55 & 50 & 25\end{array}$

$\begin{array}{llll}1 & 25 & 50 & 25\end{array}$ 2672a Variabilis. New tints, chamois, sal-
mon and terra cotta, with faces ... $25 \% 3$ Victoria, claret red.............

2679 Cardinal, beautiful bright red.......

2681 Emperor Frederick, rich velvety, marcon, with golden frilled edges.

2682a Giant Madame Perret, wine colored, penciled pink and red............

2689 Giant Curled and Ruffed, Germania or Masterpiece ..................

$2691 \frac{1}{2}$ Giant Paris, large spotted..........

2692 Giant Parisian Stained, fancy .....

2694a Giant Fancy Blotched and Striped in gay light colors _.............

2699 Giant Cassier Hultensia, bluc spots on delicate pink ground blossom pink, beautifully blotched
Cassier Giant Blotched, 3 and 5

spotted, mixed
Improved Giant Cassier Canary Bird,
5 blotched golden vellow

2702 Improved Giant Cassier, extra 5 blotched strain ................ German prize flowers. . . . . . .

2714 Giant Striped Giant Auriculæflora beautifully marked and margined

2715a Giant Bronze. Novel colar........

2716 Giant Purple, solid color ...........

2717 Giant Lilarosa, fine colors ..........

2718 Giant Blve (Emperor William) true..

2719 Giant Black Blue, fine...... . . . . . golden.

2721a Giant Rubj Red

2722 Giant Lord Beaconsfield ............

2723 Giant Fire King (fiery faces), purplish scarlet, yellow margin and golcen

2724 Giant Golden, with dark centre .... 120

2725 Giant Violet, Yellow Marbled _...... 120

2726 Giant White, dark centre ........... 110

2727 Giant Violet, White llarbled ...... 1 25

2728 Giant Violet Blue, distinct........

2728 Giant Azure Blue, new
2730 Giant Adonis, large dark margined light blue flowers, with rellowish

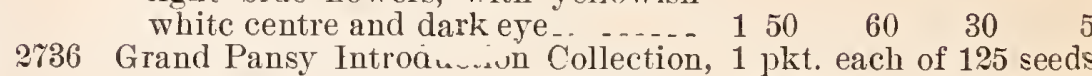

of the above 55 kinds $(6,875$ seeds, value $\$ 3.30$ ), for $\$ 1.75$.

Tr. Pkt. Tr. Pkt. Pkt.

$\begin{array}{llll}75 & 70 & 35 & 5\end{array}$

$30 \cdot 15$

$20 \quad 48 \quad 24$

$\begin{array}{llll}75 & 30 & 15 & 5\end{array}$

$\begin{array}{llll}275 & 1 & 10 & 55\end{array}$

$75 \quad 30 \quad 15$

$50 \quad 100 \quad 50$

$\begin{array}{lllll}3 & 00 & 1 & 20 & \& 0\end{array}$

$\begin{array}{llll}00 & 120 & 60\end{array}$

$\begin{array}{llll}75 & 1 & 10 & 55\end{array}$

$350 \quad 140 \quad 70$

10

$\begin{array}{lllll}00 & 120 & 60 & 8\end{array}$

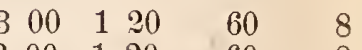

$\begin{array}{llllll}3 & 50 & 1 & 40 & 70 & 10\end{array}$

$\begin{array}{lllll}120 & 48 & 24 & 5\end{array}$

$\begin{array}{lll}00 & 80 & 40\end{array}$

$00-48 \quad 24$

$\begin{array}{lll}20 & 48 & 24\end{array}$

$\begin{array}{lll}25 & 90 & 45\end{array}$

$120 \quad 48 \quad 2$

$\begin{array}{llll}2 & 00 & 80 & 40\end{array}$

$\begin{array}{lll}80 & 40 & 6 \\ 48 & 24 & 5 \\ 48 & 24 & 5 \\ 42 & 21 & 5 \\ 50 & 25 & 5 \\ 48 & 24 & 5 \\ 80 & 40 & 6\end{array}$

$200 \quad 80 \quad 40$

$\begin{array}{llll}200 & 80 & 40 & 6\end{array}$

$\begin{array}{lllll}3 & 00 & 1 & 20 & 60\end{array}$ 
2667 Dark Wallflower, brown ............

2668 Fancy Striped, in shades

2669 Rose Irarbled or Marmorata -

2670 Snow Queen, satiny white; no eye.

2671 Pelargoniflora, beautiful markings . .

2672 Fire Dragon, or brown and gold ....

2674 Delicata, porcelain blue and white -.

2675 Steel Blue, white edged ............

26\%6 Auriculæflora, purple and bronze...

2678 Quadricolor or Rainbow ............

2680 Gloriosa, large velvety black purple, with faint faces

2682 Crown Prince, black-tinted golden bronze . . .

2683 Meteor, large, beautiful wine color --
2684 Fire King, golden yellow, with dark fiery blotches .... - ..............

2684a Coppery Scarlet, rich brown red...-

2685 Red Riding Hood or Erilliant .

2686 Prince Bismarck, brown and golden

2688 Rex, blackish, violet purple........

section 4.-PRICE LIST OF PANS KNOTN VALUE.

2632 N.Y. Giant Market Blend

2643 Red Velvet Blend, rich red shades

2644 Bedding Mixture, hardiest kinds...

2677 Fancy Silver Banded.........

2690 Extra Fine German Mixture, superior

2690a English Show Faced ...............

2691 Giant Fancy Belgian..............

2694 Giant Red and Brown Stained (Bugnots)

2695 Giant Paris Market

2696 Giant Butterfly Mixture .............

2697 Ne Plus Ultra Mixture, celebrated German strain of giant 5 blotched and stained varieties ............

2698 Giant Odier, in fine markings . 2704 French Giant Trimardeau, large flowering, extra fice, mixed.....
arge Flowering French, mixed

2705 Large Flowering French, mixed .....

2707 Giant Stained, fancy mixed.........

2707a Giant Parisian, striped
2709 New Buttercup Blend, the finest yellow strain for florists

2r10 New York Improved Fancy Show
Blend, the best high grade mixture for florists

2711 Giant Five Spotted (Roemer) ......

2712 Giant Sweet Scented ..............

2713 Royal Exhibition of Euglish Giants.

2:34 Giant Improved Trimardeau, mixed, extra

2735 Giant Trimardeau, mixed, $1 \mathrm{~b},, \$ 9.00$

2737 Prize Medal Blend, saved from exhibition plants that won prizes.. $1200 \quad 4 \quad 80 \quad 2 \quad 40$

2744 Mixed, good quality, lb., $\$ 4.00 \ldots \ldots$

2745 Tufted Pansy or Large Flowering Hybrid Violet, free flowering and sweet scented...

$200 \quad 80 \quad 40$

Papaver, see Poppy, Nos. 2921-53.

2748* Pardanthus Chinensis (Blackberry Lily) ....

2750 Passion Flower, Cœrulea, blue ..... . . . . . . .

2792* Peas, Perennial, white ..................

2793* Peas, Perennial, red

2794* Peas, Perennial, mixed -

2798* Pentstemon, finest, mixed .................

2800 Pentstemon Hartwegii (Gentianoides) Grandiflorus, newest large flowering mixed.

2804 Perilla Nankinensis Laciniatis . . . . . . . . .

\section{PETUNIA HYBRIDA.}

2808 Fine mixed, 1b., $\$ 2.50$

2809

Extra choice, mixed, lb., $\$ 4.00 \ldots \ldots$

2810

2812

Blotched and striped mixed, 1st quality....

2814 Howard's Star, rich crimson maroon with white stripes forming a star; best for borders, hanging baskets, etc............. 100

2816 Dwarf Inimitable, true

2818 Adonis, dwarf, fiery carmine, white throat.-

2820

Rosy Morn, dwarf, pink with large white

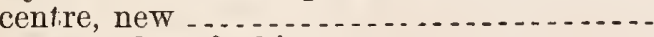

Snowball, dwarf white....................

2824

Double-flowered, mixed, 1,000 s., $50 \mathrm{c}_{\ldots} \ldots \ldots .$.
LA HA G FLO W WR ING VAIR I ETIES.

2828 Ring of Emerald, pink and crimson flowers

with green edges, 1,000 seeds, $50 \mathrm{c} \ldots \ldots . . .$.

2830 Grandiflora, single mixed, 1,000s., 50c . . . . . . .

2832 Grandiflora, double mixed, 1,00 's., $\$ 1.00 \ldots$

2834 Fringed, giant white, Snowstorm, 1,000s.,

$50 \mathrm{c}$

2836 Fringed, giant single, mixed, 1,000s., $50 \mathrm{c}$

2838 Giant of California, single, mixed, 1.000 s. $50 \mathrm{c}$

2839 Ruffled Giants, single, mixed. 1,000s., $75 \mathrm{c}$

2840 Ruffled Giants, double, mixed, 1,000s., \$1.25

Tr. Pkt Pkt.

2844 Phacelia Campanularia

20

$\begin{array}{rr}25 & 8 \\ 25 & 8 \\ 35 & 10 \\ & \\ 25 & 8 \\ 25 & 8 \\ 35 & 10 \\ & \\ 25 & 8 \\ 30 & 8 \\ 35 & 10\end{array}$

28

\section{PHLOX DRUMMONDII.}

2840 Finc mixed, 1b., $\$ 3.00$

25

10

GIA A DIFLORA, LARGE FLOUVEING.

2848 Chamoise Rose, white eyc. 2849, Crimson, white eye; 28.j0, Deep Rose; 2851, Kermesina Splendens, white eye; 2852, Purple, white eye; 2853, Scarlet Striped; 2854 Stellaria Splendens; 2855, Vivid Scarlet 2856, White; 285\%, Yellow (Isabellina); each

2858 Our own mixture of above separate colors -

2860 Large Flowering, mixed, lb., $\$ 4.00$.

$\begin{array}{lll}60 & 15 & 5 \\ 60 & 15 & 5 \\ 40 & 10 & 3\end{array}$

\section{NANA COMLACTA (for Pots).}

2863 Chamoise Rose. 2864, Fireball; 2865 Snowball; 2866, Mixed; each .... .

2867 Phlox Fringed (Fimbriata), mixed

2868 Phlox, Star of Quedlinburg (Cuspidata), mixed

2870* Phlox, Perennial (decussata), mixed; hardy garden 'Phlox; sow seed in cold frame in early winter.

2871* Phlox, Large Flowering Hybrids, mixed .

2873 Physianthus Albens (Cruel Plant)

2875* Physostegia Alba, white.

$2876^{*}$ Physostegia Virginica, rose

\section{PINKS-DIANTHUS.}

\section{DOUBLE FLOUTHING VARIETIES.}

$\begin{array}{lll}2878 & \text { Chinensis (Indian Pink), crimson } \ldots \ldots \ldots \ldots & 40 \\ 2879 & \text { Chineusis, white } \ldots \ldots \ldots \ldots & 35\end{array}$

2880 Chinensis, mixed, lb., $\$ 2.00 \ldots \ldots \ldots \ldots$

2881 Imperialis (Imperial Pink), dwarf, pure white

2882 Imperialis, Fireball, dwarf fiery scarlet

2883 Imperialis, dwarf, mixed

2884 Imperialis, extra fine, mixed, 1b., $\$ 2.50$

2885 Heddewigii (Japan Pink), Mourning Cloak dark purple, margined white, clove-scented

2886 Heddewigii Diadematus (Diadem Pinks), mixed

Oz. Tr. Pkt. Pkt.

$\begin{array}{rrr}50 & 1 \tilde{5} & 3 \\ 25 & 10 & 3 \\ 20 & 10 & 3 \\ 10 & 5 & 3 \\ 10 & 5 & 3 \\ 25 & 10 & 3 \\ 50 & 20 & 5 \\ 10 & 5 & 3\end{array}$

2887 Heddewigii Stellaris, Star Pinks, mixed..... Royal Pinks

$2 \$ 89$ Heddewigii, Snowball, pure white

2890 Heddewigii, mixed

2892 Laciniatus (Fringed Pinks) Salmon Kin

2893 Laciniatus Alba, white fringed .

2894 Laciniatus, mixed .

$2895^{*}$ Plumarius (Florist or Grass Pinks), mixed

2896* Plumarius, Dwarf Erfurt, early flowering, extra ....

\section{SINGLE WLOWERING VARIETIES,}

$\begin{array}{rrr}20 & 5 & 3 \\ 40 & 10 & 3 \\ 40 & 10 & 3 \\ 75 & 20 & 3\end{array}$

2899 Heddewigii, Queen of Holland, white.

2901 Heddewigii, Eastern Queen

2902 Heddewigii, Nobilis (Royal Pinks), mixed --

2903 Heddewigii, Stellaris, Star Pinks, mixed.

2904 Heddewigii, The Bride, white with purplish

Heddewigii, mixed

Laciniatus, Salmon Queen

Laciniatus, Snowflake, white fringed.-
Laciniatus, new dwarf hybrids, mixed..

Laciniatus, fringed, mixed...
15

10

15

1.

10

10

15 
2911* Plumarius (Pheasant's Eye Pink), 1b, 75c 2912* Plumarius, Cyclops or Scotch Pinks, mixed. 2913* Plumarius, Semperforens (Clove Pinks)

2914* Platycodon Grandiflorum, mixed _... . . _. _ 25

2917* Polyanthus, fine mixed .................. 125

2918* Polyanthus, gold-laced, mixed _......... 150

2920* Polemonium (Jacob's Ladder), blue, 1b., $50 \mathrm{c}$.

10

\section{POPPY.}

\section{SINGLE FLOWERING ANNUAL SORTS.}

2921 Danebrog, brilliant scarlet with white spots 10

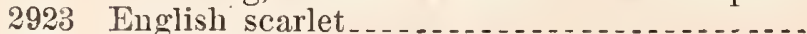

2924 Fire Dragon (Lævigatum)

2926 Glaucum (Tulip Poppy), deep scarlet ...... -

2927 Maid of the Mist, large white with fringed petals

2928 Miss Sherwood, new; chamoise-rose ......

2929 Peacock, brilliant scarlet with glossy black

2930 Shing

2931 Shirley, dwarf mixed

2932 The Bride, pure white

2933 Umbrosum, vermillion with black spots . . . .

2934 Mixed

DOUBLE FLOWIERT ANNUAL SOR'TS.

2937 American Flag, white with scarlet _........ 15

2938 Fairy Blush, white and rose ............. 15

2940 Golden Leaved, new ; scarlet flowers, yellow foliage

2941 Mikado, white, striped crimson

2942 White Swan .............

2913 Carnation-flowered, striped and mottled, mixed ....

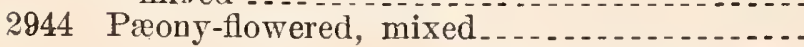

\section{PERENNIA L VARUETIES.}

2948* Bracteatum, large flowering scarlet......... . 2949* Nudicaule (Iceland Poppy), double scarlet... 150 2950* Nudicaule Hybridum, new .............. 250

2951* Nudicaule, Mixed

$2952 *$ Oriental, dark red

2953* Oriental, new hybrids, mixed -

Poppy, Prickly, see Argemone, No. 1570.

Poppy, California, see Ésch. Nos. 2n84-88.

2968 Portulaca, large flowering single mixed, lb. $\$ 2.00$

2972 Portulaca, large flowering double, white....

2974 Portulaca, large flowering double, mixed ...

2976 Portulaca, fine double, mixed ............. 150

2980* Potentilla, single hybrids mixed

Pot Marigold, see Calendula, Nos. 1794-79.

Primrose Evening, see Oenothera, No. 2556.

\section{PRIMULA (Primrose).}

2981* Cachemiriana, rosy lilac, early flowering . . 2982* Cortusoides, rose, oz $\$ 1.25$

2983* Cortusoides, Sieboldi, large flowering mixed 2983e Floribunda, yellow, winter flowering...... 2983k Forbesi, "Baby Primrose," rosy lilac, $\frac{1}{16} \mathrm{oz}$. $\$ 1.25$

2984 Verticillata Grandiflora (Abyssinian Primrose), large white powdered leaves, golden yellow fuwers, fine for forcing

2985 Mollis, rare and beautiful forcing species...

\section{OBCONICA VARIETIES.}

2986 Obconica, pale lilac (type), $\frac{1}{16} \mathrm{oz} ., 75 \mathrm{c} \ldots \ldots$ 2986e Flora plena, double flowering............

2986 g Grandiflora, Oculata, new

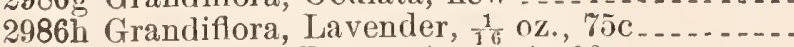
2986 i Grandiflora, Rosea, $\frac{1}{16}$ Oz. \$1.00

2986 j Grandiflora, Violacea, $\frac{1}{16} \mathrm{OZ} ., \$ 1.00$

$2986 \mathrm{k}$ Grandiflora, Alba, $\frac{1}{16} \mathrm{Oz} ., \$ 1.00 \ldots$

2986n Grandifllora, Hybrida, fine mixed, $\frac{1}{16}$ oz., 60

2986r Grandiflora, Compacta, new

2987 Grandiflora. Fimbriata, large fringed, perpetual flowering, $\frac{1}{16}$ oz., $\$ 1.00$

17a Grandiflora, Fimbriata, Alba, white, $\frac{1}{16}$ o $\%$.
10

$2987 \mathrm{~b}$ Grandiflora, Fimbriata, Rosea, $\frac{1}{15}$ oz., \$1.50

$2987 \mathrm{c}$ Grandiflora, Fimbriata, Violacea, $\frac{1}{16}$ oz. , $\$ 1.00$

$\begin{array}{lrr}1000 s & \text { Pkt. Tr. } & \text { Pkt. } \\ 50 & 15 & \tilde{5} \\ 50 & 15 & 5\end{array}$

2938 Primula Sinensis (Chinese Primrose), mixed, small flowered (type), oz, $\$ 3.50 \ldots \ldots$ Primula Elatior, see Polyanthus, Nos. 291

STAR (Stellata) PRIMULAS.

2989e Giant white ............................... 2989f King of the Star, deep carmine . . . . . . . . . 2989 g Salmon-pink

$2989 \mathrm{~h}$ Finest mixed

$25 \quad 10 \quad 5$

SINGLE LARGE FLO WERING FRINGED PRIMULAS.

2992 Alba, pure white, $1 / 8$ oz., $\$ 2.00 \ldots \ldots \ldots \ldots . \ldots 100 \quad 25 \quad 10$

2993 Globosa Alba, fine white................. 125 . $30 \quad 10$

2994 Rosea, bright rose, 1/8 oz.,\$2.00_...... $100 \quad 25 \quad 10$

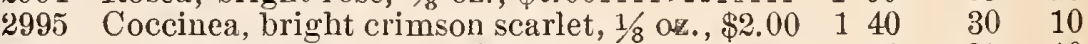

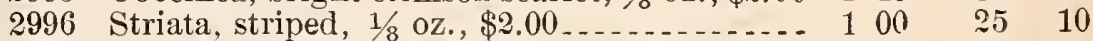

2997 Punctata Elegantissima, crimson spotted _... $140030 \quad \begin{array}{llll}4 & 40\end{array}$

2998 Kermesina Splendens, crimson yellow eye, $1 / 8$ oz. $\$ 1.75 \ldots \ldots \ldots$. $100 \quad 2510$

3000 Alba Magnifica, magnificent white, $1 / 8$ oz., $\$ 2.25 \ldots . . . . . . . .1825$

3001 Mont Blanc, large, milky white, 1/8 oz., $\$ 2.25$

3002 Coerulea, clear sky blue, $1 / 8$ oz., $\$ 3.00 \ldots \ldots$

3003 Chiswick Red, bright red, 1/8 oz., $\$ 2.25 \ldots$

3004a Best Imported Mixture, oz., $\$ 12.00 \ldots \ldots \ldots$.

3004 b New Giant flowering white, $1 / 8 \mathrm{oz} ., \$ 4.00 \ldots$

$3004 \mathrm{c}$ New Giant flowering red, $1 / 8$ oz., $\$ 4.00 \ldots \ldots$

$125 \quad 30 \quad 10$

$\begin{array}{llll}1 & 25 & 30 & 10\end{array}$

$\begin{array}{llll}1 & 50 & 35 & 10\end{array}$

$\begin{array}{lll}125 & 30 & 10\end{array}$

$\begin{array}{llr}50 & 15 & 5\end{array}$

$\begin{array}{lll}100 & 25 & 10\end{array}$

$200 \quad 50 \quad 10$

SINGLE LARGE FHOWERING FRINGED FERN-LEA VED PRTRULAs.

(Macrophylla Filicifolia Fimbriata.)

3006 Pure white, $1 / 8$ oz., $\$ 2.00 \ldots \ldots \ldots \ldots \ldots \ldots$. $100 \quad 2510$

3008 Bright red, $1 / 8$ oz., \$2.00

3010 Choicest mixed, 1/8 oz., $\$ 1.50 \ldots \ldots \ldots \ldots \ldots 20 \quad 75 \quad 20$

\section{DOURLE LARGE HLO IW GRING FRINGED PRINULAS.}

3012 Pure white

$\begin{array}{ccc}1.00 \mathrm{cs} . & 100 \mathrm{~s} . & \text { Pkt } \\ 2 & 30 & 30\end{array}$

301: Bright red

$\begin{array}{llll}2 & 50 & 30 & 10\end{array}$

3014 Iixed, from prize flowers $\begin{array}{llll}200 & 25 & 10\end{array}$

3017 N. Y. special Florists' Blend. This is our own mixture of all the above kinds, commencing with No. 2992. Recommended to those who require a large assortment, and do not grow separate colors, $1 / 8$ oz., $\$ 3,75 \ldots$

3026 Pueraria Thunbergiana (Kudzu Vine) ....... 3028* Pyrethrum, Golden feather, yellow foliage

3029 Pyrethrum Selaginoides, moss-like yellow foliage.

3030 Pyrethrum, Golden Gem, double white....

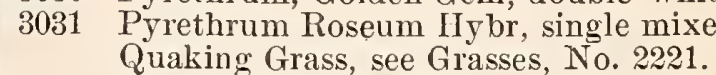

Ragged Robin, see Lychnis, No, 2394.

Red Hot Poker, see Tritoma, No. 3249.

Reed, see Grasses, Nos. 2230-31.

Reseda Odorata, see Mignonette, Nos. 2436-49.

Rhodanthe Maculata, see Everlastings, No. 2123

RICINUS (Castor Oil Bean).

Add $\&$ c. to the price when ordered sent by mail.

$\begin{array}{lll}50 & 20 & 5 \\ 50 & 20 & 5\end{array}$

3040 Best mixed

3041 Borboniensis Arboreus...

3042 Cambodgensis, very dark-leaved ..........

3043 Gibsonii, dark red foliage ................

3044

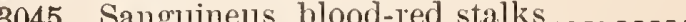

3046 Tricolor Magnificus . . . . . . .

3047 Zanzibaricnsis mixed ... ... . . . . . . . . . .

Oz. Tr.Pkt. Pkt.

$\begin{array}{lll}85 & 20 & 5 \\ 20 & 10 & 3\end{array}$

$40 \quad 10 \quad 3$

2510

$50 \quad 15 \quad 3$

$50 \quad 20 \quad 10$

$3050 *$ Rocket Sweet, mixed.

3052* Romneya Coulteri (Cali-Tree Poppy) Rose Campion, see A grostemma, No. 1509.

3059* Rose Polyantha Multiflora Nana, blooms the

Lb. $1 / 4$ b. ()z

$\begin{array}{lll}60 & 20 & 10\end{array}$

$\begin{array}{lll}00 & 35 & 10\end{array}$

$60 \quad 20 \quad 10$

$60 \quad 20 \quad 10$

$\begin{array}{lll}60 & 20 & 10\end{array}$

$70-25 \quad 10$

Oz. Tr. Pkt, Pkt,

$10 \quad 5 \quad 3$ first season from seed, 100 berries, $25 \mathrm{c}_{\ldots} \ldots$ 
3061 Rudbeckia, Bicolor Superba

3062 Rudbeckia, Bicolor Superba Semiplena.....

3063* Rudbecka Laciniata _._.

3069 Saintpaulia Ionantha, small blue perpetualflowered green house plant, $1,000 \mathrm{~s}$., $50 \mathrm{c} \ldots$..

3072 Salpiglossis, large flowering mixed .........

3073 Salpiglossis Emperor mixed, new, very large flowering

SALVIA.

3076 Coccinea Splenden

3077 Splendens, scarlet.

\section{SALVIA.}

3078 Splendens, Lord Fauntleroy, the only dwarf Salvia . . .

3079 Splendens Silverspot, yellow spotted foliage 3080* Argentea, white leaved

3081 Patens, deep blue, 100 s., $30 \mathrm{c}$

3083* Santolina Maritima, white leaved

3085 Sanvitalia Procumbens, double

3087 Saponaria (Calabrica), red

3088* Saponaria Caucasica, fl. pl

SCABIOSA (Mourning Bride).

3090 Large flowering double, snow white, $1 \mathrm{~b} ., \$ 1.50$

3091 Large flowering double, flesh-pink ..........

3092 Large flowering double, fiery scarlet. ......

3093 Large flowering double, king of the blacks. -

3094 Large flowering double, lilac . - . . . . . . . . - .

3095 Large flowering double, mixed, lb., $\$ 1.25 \ldots$

3096 Sncwball, double pure white, 1b., $\$ 1.00 \ldots$

3097 Major, double mixed 1b., $75 \mathrm{c}_{\ldots} \ldots \ldots . . .$.

3098 Dwarf, double mixed, lb., $\$ 1.00 \ldots \ldots \ldots \ldots$

3099* Caucasica, lavender ...................

3100* Caucasica Alba, white........................

$3101^{*}$ Japonica, a new hardy perennial from Japan with ragged-edged flowers 2 inches across _

Scarlet Sage, see Salvia, Nos. 3076-79.

3102 Schizantluus, best mixed, lb., $\$ 1.00 \ldots \ldots \ldots$.

3103 Schizanthus Wisetonensis, new ............

Sea Lavender, see Statice. No. 3124

3105 Sedum Cœruleum (blue Stonecrop) .........

3107 Senecio Elegans, double mixed ..................

3108 Sensitive Plant, $1 b ., \$ 2.25 \ldots \ldots \ldots \ldots \ldots$

Shasta Daisy, see Daisy, No. 2039.

3112 Silene Pendula, lb., 40c.............

Silene Armeria, sec Catchfly, No. 1861.

Silk Oak, see Grevillea, No. 2254.

3116 Smilax, fresh seed, lb., \$2.50

3117 Smilax, Myrtle-leaved, a fine decorative novelty, pkt. 10 seeds ...................... Snapdragon, see Antirrhinum. Nos. 1543-52.

3120 Solanum Capsicastrum (Jerusalem Cherry) -

3121 Solantm Eeaforthianum, rapid growing climber constantly covered with lilac-blue Spider Plant, see Cleome, No. 1948.

3124* Statice Incana Hybrida Nana, mixed (Sea

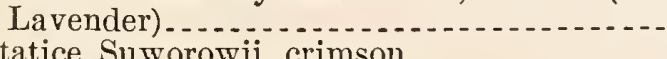

3126 Statice Suworowii, crimson

3129 Stevia Serrata, white .................. 50

\section{STOCKS, GERMAN 10 WEEKS.}

\section{LARGE FLO WWRING DWART.}

3140 White. 3141 Blood red; 3142, Bright rose;

3143, Canary yellow; 3144, Crimson; 3145,

Light blue; 3146 , Our own mixture; each.

3147 Fine mixed as imported

$150 \quad 20 \quad 5$

\section{CUT-A ND.COME.AGA IN .}

3150 Princess Alice, white. 3151; Blood red; 3152, Brilliant rose; 3153 , Canarv vellow 3154, Dark blue; 3155, Light blue; 3156 ,

Our own mixture; each............... 200

3157 Fine mixed as imported................... 150

25

GARLY FLOW ERING WINTER.

3159 Beauty of Nice, daybreak pink ........... 400

3160 Queen Alexandra, delicate, rosy lilac, $1 / 8 \mathrm{oz}$, $\$ 1.00$

3161 Snow-white

3162 Souvenir de Monaco, carmine-red, very large

flowering

3163 Empress Elizabeth, blood-red.

VARIOCS SOR'TS.

3164 Snowflake, white forcing ............... $400_{00}^{\text {Uz. }}{ }_{25}{ }_{10}$

$\begin{array}{llllll}3166 & \text { Emperor, large flowering mixed.......... } & 3 & 50 & 25 & 10\end{array}$

3168 Dwarf German mixed _................... $50 \quad 50$

3169* Stokesia Cyanea (Cornflower Aster), lg. flg.

blue, very beautifui ................

3171 Streptocarpus Wendlandii, 1,000 s., 50c $\ldots$

3172 Streptocarpus, large flowering new hybrids,

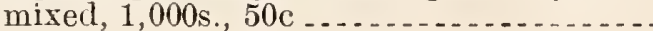

$\begin{array}{lll} & 25 & 5 \\ - & 25 & 5 \\ - & & \\ - & 25 & 5\end{array}$

\section{SUNFLOWER.}

3174 Californicus, double, lb., $75 \mathrm{c}$

3175 Cucumerifolius, small-flowered miniature

3176 Cucumerifolius, Orion, twisted petals ......

3177 Cucumerifolius, Double and semi-double ....

3178 Globosus Fistulosus, 1b., 70c $\ldots$

3179 Dwarf double, lb., $\$ 1.00$

3180 Dwarf single, $1 \mathrm{~b} ., 60 \mathrm{c}$

$\begin{array}{rrr}10 & 5 & 3 \\ & & \\ 10 & 5 & 3 \\ 15 & 5 & 3 \\ 75 & 15 & 5 \\ 10 & 5 & 3 \\ 10 & 5 & 3 \\ 10 & 5 & 3\end{array}$

Swan River Daisy, see Brachy., No. 1750.

\section{SWEET PEAS.}

When ordering by the $1 \mathrm{~b}$. to be sent by mail, add $8 \mathrm{c}$. per $1 \mathrm{~b}$. to cover postage.

3186 America, blood-red, striped white

3187 Apple-blossom, bright pink and blush .....

3188 Blanche Ferry, Extra Early, pink and white

Captain of the Blues, purple and lavender

3190

3191

3192

3193

Coccinea, very bright cerise

Countess of Iogan, bright blue....

3194 Dorothy Eckford, the finest giant white

10

53

3195

Dorothy Tennant, rich rosy mauve...

3197 Earliest of All, a week earlier than Blanche Ferry

3199 Gorgeous, deep orange-salmon

3200 Her Majesty, Jarge, beautiful soft rose......

3201 Hon. Mrs. E. Kenyon, delicate primroseyellow, larger than Mrs. Eckford.

3202 Katherine Tracy, soft brilliant pink

3203 Lady Grisel Hamilton, light lavender -

3204 Lottie Eckford, white, suffused and edged with lavender

3205 Mars, large, deep scarlet ...................

3206 Miss Willmott, rich, deep orange-pink, very large ............... 3207 Mont Blanc, new; earliest flowering white

3208 Mrs. Dugdale, large deep rose.............

3209 Mrs. Eckford, primrose-yellow.

3210 Navy Blue, new, deep violet-blue

3211

3212

3213

3214

3215

3217

3218

3219

3220

Pink Friar, white, watered rosy crimson....

Royal Rose, deep rosy pink ................

Salopian, beautiful brilliant scarlet ..........

Eckford's Hybrids, mixed. .

Fine mixed

Giant California, mixed

Our own special mixture containing all the finest large flowering varieties . ...........

Double mixed, about one-third come double.
New Orchid-flowering, Countess Spencer .. Cupid, dwarf, finest mixed...

Sweet Sultan, see Centaurea, No. 1888.

$\begin{array}{rrr}\text { Lb. } & \text { 1/4 Lb. } & \text { Oz. } \\ 25 & 10 & 5 \\ 25 & 10 & 5 \\ 25 & 10 & 5 \\ 25 & 10 & 5 \\ 25 & 10 & 5 \\ 25 & 10 & 5 \\ 25 & 10 & 5 \\ 25 & 10 & 5 \\ 50 & 20 & 10 \\ 25 & 10 & 5 \\ 25 & 10 & 5 \\ & & \\ 30 & 10 & 5 \\ 25 & 10 & 5 \\ 25 & 10 & 5 \\ 25 & 10 & 5 \\ & & \\ 25 & 10 & 5 \\ 25 & 10 & 5 \\ 30 & 10 & 5 \\ & & \\ 25 & 10 & 5 \\ 25 & 10 & 5 \\ & & \\ 30 & 10 & 5 \\ & & \\ 25 & 10 & 5 \\ 25 & 10 & 5 \\ 25 & 10 & 5 \\ 25 & 10 & 5 \\ 25 & 10 & 5 \\ 25 & 10 & 5 \\ 25 & 10 & 5 \\ 25 & 10 & 5 \\ 20 & 10 & 5 \\ 25 & 10 & 5 \\ & & \\ 30 & 10 & 5 \\ 30 & 10 & 5 \\ 00 & 35 & 10 \\ 35 & 10 & 5 \\ & & \end{array}$

\section{*SWEET WILLIAM.}

3226

3227

3228

\begin{tabular}{ll|l}
25 & 10 & 32230
\end{tabular}

$\begin{array}{llll}2 \overline{50} & 25 & 10 & 3231\end{array}$

3232

\begin{tabular}{ll|l}
25 & 10 & 3233 \\
\hline & 10 & 3234 \\
\hline
\end{tabular}

25

3234
Single, white, lb., $\$ 1.00$

Single, blood red, 1b., $\$ 1.00 \ldots$

Single, mixed, lb., $65 \mathrm{c} \ldots \ldots \ldots$

Mammoth, single, mixed ................

Mammoth, double, mixed ................

Mammoth, Holborn Glory ................

Double, crimson

Double, white

$\begin{array}{rrr}\text { Oz. } & \text { Tr.Pkt. } & \text { Pkt. } \\ 10 & 5 & 3 \\ 10 & 5 & 3 \\ 10 & 5 & 3 \\ 15 & 5 & 3 \\ 40 & 10 & 3 \\ 30 & 10 & 3 \\ 125 & 20 & 5 \\ 40 & 10 & 3 \\ 25 & 10 & 8\end{array}$


3235* Srmphyantra Hofmannii

3237 Tecoma Smithii dwarf erect bush, constantly corered with lemon and dark orange flowers

3241* Thermopsis Fabacea

3243 Thunber ria mixed

3245 Torenia Bailloni, golden Jellow, $\frac{1}{16} \mathrm{oz}$, $50 \mathrm{c}_{-}-$

3246 Torenia Fournieri, blue, Jellow throat . . . . -

$324 i$ Torenia Fournieri, White Tings

Tree Cypress, see Ipomopsis, No. 2333.

3249* Tritoma Uvaria Nobilis

Tropæolum, see Nasturtium, Nos. 2464-2524.

Tropæolum Peregrinum, see Canary Vine. No. 1810.

$3252 \div$ Tunica Saxifraga...

$3254 *$ Taleriana, mixed

\section{VERBENA HYBRIDA.}

3260 Mammoth, Pink shades. 3261, Purple shades. 3262, Pure white; 3263, Scarlet shades: 3264. Striped shades: 3265, IIixed: each..

3266 Candidissima, pure white ...............

3267 Dark blue, white eye

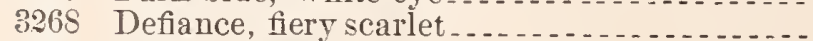

3269 Fancy striped.

$32{ }^{2} 0$ Lutea jellow

3271 Scarlet, white eye

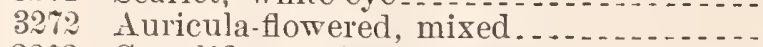

32'73 Grandifiora, mixed

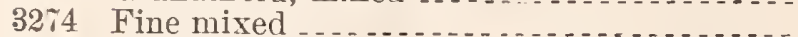

3276 Verbena Erinoides (Ioss Verbena) white....

3277 Verbena Erinoides, purple ...............

32 is Verbena, Sweet-scented (Teucrioides) ........

3279 Verbena Tenosa, rich purple, fine bedder...

Terbena Citriodora, see Lemon Verbena, To. 2356 .

\begin{tabular}{ccc} 
Oz. & \multicolumn{2}{c}{ Tr. Pkt. Pkt. } \\
25 & 10 & 3 \\
& & \\
50 & 25 & 5 \\
$-\overline{4}$ & 25 & 5 \\
40 & 10 & 3 \\
$\overline{75}$ & 25 & 5 \\
00 & 20 & 5 \\
& 25 & 5 \\
00 & 20 & 5 \\
& & \\
& & \\
15 & 5 & 3 \\
15 & 5 & 3
\end{tabular}

3282* Veronica, mixed

3284 Vinca Rosea, mixed

Virginian Stock, mixed, lb. $40 \mathrm{c}$

3292 Wallflower, single, mixed, 1b., $\$ 1.00 \ldots \ldots$

3293 Wallflower, single, Parisian forcing, blooms first season . . . . . Pa . . . . . . . . .

3294 Wallflower, Goliath, fine for forcing ... . . .

3295 Wallflower, double mixed........................

3298 Whitlavia Grandifiora, mixed -

3300 Wimandia Vigieri .

3301 Wild Cucumber

WT istaria Sinensis, blue

Wood Fringe, see Adlumia, No. 1493.

Woodruff, see Asperula, No. 1590.

Xeranthemum, see Everlastings. No. 2124.

Youth and Old Age, see Zinnias, Nos, 33310-24.

3304* Yucca Filamentosa ........

Zebra Grass, see Grasses, No. 2239.

\section{ZINNIA.}

$\begin{array}{lll}00 & 20 & 5 \\ 50 & 15 & 3 \\ 60 & 15 & 3 \\ 60 & 15 & 3 \\ 60 & 15 & 3 \\ 60 & 15 & 3 \\ 50 & 15 & 3 \\ 50 & 15 & 3 \\ 60 & 15 & 3 \\ 35 & 10 & 3 \\ 50 & 15 & 3 \\ 35 & 10 & 3 \\ 25 & 10 & 3 \\ 25 & 10 & 3\end{array}$

3310 Single, mixed

3311 Zebra, striped

3312 Curled and 0

3313 Fringed, double mixed

3314 Tall double mixed

3315 Mammoth-fiowering, white (Queen Victoria)-

3316 Iammoth-flowering, mixed _...........

3317 Dwarf double, canary .......................

32:18 Dwarf double, orange.

3319 Dwarf double, scarlet

3320 Dwarf double, white

3321 Dwarf double, mixed ...................

3322 Pompone, mixed

3323 Tom Thumb, double mixed .............

3324 Haageana Hybrida, double ixed ...........

$\begin{array}{lll}25 & 10 & 3 \\ 50 & 15 & 3 \\ 40 & 10 & 3 \\ 45 & 15 & 3 \\ 20 & 10 & 3 \\ 75 & 15 & 3 \\ 40 & 10 & 3 \\ 35 & 10 & 3 \\ 35 & 10 & 3 \\ 35 & 10 & 3 \\ 35 & 10 & 3 \\ 25 & 10 & 3 \\ 25 & 10 & 3 \\ 50 & 15 & 3 \\ 75 & 15 & 3\end{array}$

\section{VEGETABLE SEEDS.}

Packets.-We will supply all kinds of regetable and herb seeds in packets @ 3c. eaeh, except where otherwise noted. Also $\frac{1}{3}$ pint paekages of Peans, Corn ard Peas @ ic. eaeh, prepaid, or õc. not prepaid.

Delivery.-Seeds in ounees and $\frac{1}{4}$ lbs., we prepar to any post office. When ordered by the lb. or qt. to be sent by mail, add at the rate of 8 e. per $1 b$. and $15 \mathrm{c}$. per qt. to cover postage.

At prices quoted, seeds by the lb., quart, peek and bushel will be shipped by Express, Freight or Steamer, charges to be paid by purchaser. No charges made for bags or packing.

\section{ARTICHOKE}

French Green Globe. $\begin{array}{ccc}\mathrm{Lb} . & 1 / 1 \\ 200 & 60 & \mathrm{Lz} .\end{array}$

\section{ASPARAGUS.}

Barr's Nammoth: very large, early

Columbian Nammoth Thite; produces pure white

shoots requiring no artificial blanching ..... .

Conorer"s Colossal; the old standard .

Palmetto (true); large, light greell shoots; very early

$50 \quad 15$

$\begin{array}{ll}30 & 10 \\ 45 & 15\end{array}$

\section{BEANS, DWARF OR BUSH.}

\section{GREEY-PODDED VIRIETIES.}

Dwarf Horticultural; late and productive; showy pods.......... harāy . .

Extra Early Refugee; two weeks earlier than

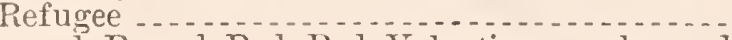
Improred Round Pod Red Yalentine; early and popular

Long Yellow Six Weeks

Refugee or Thousand to One; medium early and rery productive........................

Stringless Green Pod; extra early variety ......

Giant Stringless Green Pod Valentine; new; earlier and larger than Red Valentiue ............

\section{$\begin{array}{llll}00 & 1 & 15 & 18\end{array}$}

$\begin{array}{lllll}4 & 00 & 1 & 15 & 18\end{array}$

$\begin{array}{lllll}4 & 00 & 1 & 15 & 18\end{array}$

$\begin{array}{lllll}4 & 00 & 1 & 15 & 18\end{array}$

$\begin{array}{lllll}6 & 00 & 1 & 65 & 22\end{array}$

$\begin{array}{lllll}6 & 25 & 1 & 75 & 23\end{array}$
WAX-PODIED VARIETIES.

Currie's Rust-proof Black Max

Davis' White Kidney Wax Golden Wax

Golden-Eyed Wax

Grennell's Improved Rust-proof Golden Wax .... Improved Prolific Blaek Wax..................

Improved Stringless Refugce Wax, new . . . . . . . .

New Round-Pod Kidney ITax ................

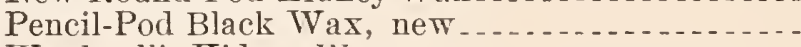

Wardwell's Kidney Wax . . . . . . . . . . . . . .

BEANS, BUSH LIMA.

Burpee's Bush Lima .... . . . . . . . . . . . . . . .

Dreer's Bush Lima

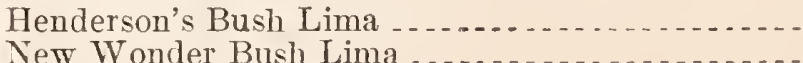

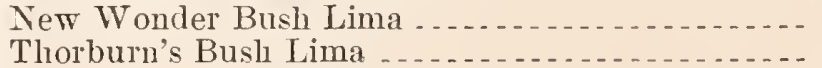

BEANS, POLE OR RUNNING.

Dutch Case Krnife .......................... 525145

Early Golden Cluster Wax...................

Horticultural or Speckled Cranberry ............ 5 2i 145

Scarlet Runner........ 50150

Large White Lima.............

Kin of the Garden Lima

Ford's Mammoth Podded Lima

Dreer's Improved or Challenger Lima........... 600006

20

\section{BEETS.}

Arlington

Bastian's Blood 'Turuip

Columbia or Carter's Excelsior

Crosby's Improved Egyptian . . . . . . . . . . . . . . .

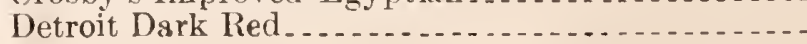


Dewing`s Improved Blood Turnip

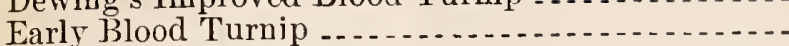

Early Flat Bassano

Improved Early Eclipse . . . . . . . . . . . . . . . . .

Lentz Blood Turnip

Long Blood Red

IINGEL-WUIRZELAND SUGAR HEETS.

Champion Yellow or Orange Globe........... . .

Golden Tankard

Red Globe, Large. . . . . . . . . . . . . . . . . . . . . . .

Lane's Imperial Sugar

Vilmorin's Improved White Sugar

BROCCOLI.

Early Purple Cape; large, compact heads of a brownish purple color; hardier than cauliflower

BRUSSELS SPROUTS.

Long Island Improved; the favorite in New York

Improved Half Dwarf or Paris Market; a sure cropper

\section{CABBAGE.}

Early Jersey Wakefield; well known popular early variety - . . Wakefield; larger and later than Jersey Wakefield. . . . . . . . . . . . . . . . . .

All Head Early; one of the best early varieties....

Early Spring; round, flat heads

Early Winnigstadt; cone-shaped heads; good for either early or late use

Early Flat Dutch; a sure header; second early -.--
Early Summer;large, round, compact heads; popular

Al! Seconons; large, quick growing varieties; good keeper

Succession; large, flat heads; fine second early
variety

surehead; late, sure header and a good keeper....

Autumn King; large, solid heads ................ market or family use ...................

Large Late Drumhead; favorite winter variety -.--
Perfection Lrumhead Savoy; the best Savoy cabbage grown

Mammoth Rock Red; largest, sure heading, red variety

CARROT.

Chantenay Half-Long (Stump-rooted) .............

Danvers Half-Long -................................

Early French Forcing Horn . . . . . . . . . . . . . . .

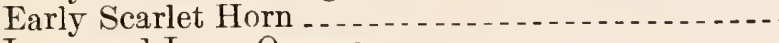

Improved Long Orange.

Nantes Half-Long Scarlet (Stump-rooted) . . . . . . . .

Oxheart or Guerande (Stump-rooted) ..............

Saint Vallery or Intermediate Red.

Large White Beloian (For cattle feeding) .........

Large Tellow Belgian (For cattle feeding).........

\section{CAULIFLOWER.}

Danish Dry Weather, new, lb; $\$ 30.00 \ldots . . . . . .$. Extra Early Dwarf Erfurt, true, lb., $\$ 20.00 \ldots \ldots$

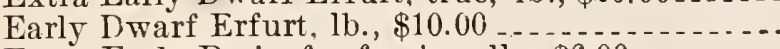
Extra Early Paris, for forcing, 1b., $\$ 6.00 \ldots \ldots$ Gilt Edge Snowball, lb., $\$ 20.00 \ldots \ldots$

Large Late Algiers, lb., $\$ 6.00 \ldots . . . . . \ldots \ldots$

Veitch's Autumn Giant, 1b., $\$ 5.00$

\section{CELERY.}

N. Y. Improved Golden Self-Blanching; extra select product tested seed. The strain planted so largely in vicinity of New York ............

Golden Self-Blanching; new crop not tested .....

Rose-Ribbed Paris Self-Blanching or Golden Rose similar to Golden Self-Blanching excepting color which is pink.

10

\section{Country Gentleman; small ears, with very deep} Egyptian or Washington Market; large ears; fine for canning Late Mammoth; the largest and latest variety ; of superior quality ....................... Stowell's Evergreen; the standard for quality -...very sweet.

\section{CRESS.}

Extra Curled or Pepper Grass................. ${ }_{25}^{25}{ }^{1 / 41 b} 10$

\section{CUCUMBER.}

\section{Early Cluster}

Early Frame or Short Green

5 Extra Early Green Prolific or Boston Pickling .....

5
10

10
5
5

Improved Long Green . . .

Japanese Climbing

Jersey Pickling

Nichol's Medium Green

White Spine, A rlington Improved .....

White Spine, Bennett's Improved

White Spine, Evergreen or Extra Long -.....-.-

White Spine, Improved Early . . . . . . . . . . . . White Spine, Peerless

$\begin{array}{lllll}5 & 00 & 1 & 35 & 40\end{array}$ $250 \quad 75 \quad 20$

West India Gherkin ur Burr

\section{DANDELION.}

Improved Thick Leaved ........................

\section{CHERVIL.}

\section{CHICORY.}

$45 \quad 15$



New York Improved Purple, Spinelcss ...........
Black Beauty; new; hardier and thriftier than any other :

Black Pekin; large, round, glossy, jet-black fruit.Early Long Purple

Large Round Purple.

\section{ENDIVE.}

Broad Leaved Batavian(Escarolle); sweet and crisp; for fall and winter......................

French Moss Curled; compact growth; finely cut leaves Green Curled; standard fall and winter variety....

90

100

90

90

\section{KALE OR BORECOLE.}

Dwarf Green Curled Scotch; grown largely in the Improved Siberian (German Greens or Sprouts)...

\section{KOHL-RABI,}

\section{OR TURNIP.POOTED CABBAGE.}

Early White Vienna; flesh tender and white...... 150 Purple Vienua; a little later than the above ...... 150

\section{IEEK.}

Broad American Flag; popular with gardeners ... _ 1 Large Carentan Winter; extra large aud hardy; good keeper

London Flag; the oldest variet

Big Boston; excellent for cold frames or open ground; large, solid heads _....... . . . . . . White-Seeded Tennis Ball or Boston Market; a popular forcing variety -

Black-Seeded Tennis Ball; heads hard, crisp and tender

California Cream Butter; large, solid heads; excellent summer variety .

Deacon; large, cabbage lettuce.................

Denver Market; large, solid, curly heads; good for "orcing or out-door planting.

Early Curled Silesia; very tender; yellowish green leaves

Early Curled Simpson; an old standard..........

Black-Seeded Simpson; large heads

Early Prize Head; large, loose heads, tinged red; crisp and tender

Golden Queen; solid golden yellow heads

Grand Rapids; fine for forcing

Hanson Improved; very large, handsome heads of fine quality

Iceberg; new; handsome, large, solid heads slightly

Improved Perpetual; best family sort; remaining a long time fit for use

Large Yellow Butter; yellow seed; tender and crisp heads; very tender

Maximum or Immensity; the largest head lettuce; very tender and crisp

Mignoneite; very solid, compact heads; russetcolored

Salamander; fine compact heads which resist summer heat...

Sensation; large, solid heads of fine quality; the best all-year-round

The Morse; white seeded; an improvement on BlackSeeded Simpson

Trianon Self-Closing Cos; the lettuce so popular in Europe

Express Cos; claimed to be the best

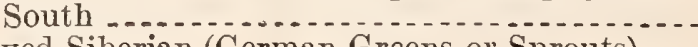

\section{LETTUCE.} tinged with red $\ldots \ldots \ldots \ldots$

Champion Market; fruit nearly round, netted; green flesh Cosmopolitan; round fruit, densely netted; very sweet, green flesh . . . . . . . . . . . . . . . . . . . . . .

Emerald Gem; early small fruit; flesh salmon color, thick and delicious . . . . . . ............

Extra Early Improved Hackensack; ten days earlier than the old Hackensack . . . .

Jenny Lind; extra early, small, green-fleshed variety
Long Island Beauty; of Hackensack type, but of superior quality netted; flesh deep salmon pink, thick and very sweet . . . . . . . . . . .

Montreal Green Nutmeg; very large, sometimes weighing 18 to $20 \mathrm{lbs}$; thick, light green flesh

Netted Gem; very early, small, green fleshed variety

Paul Rose or Petoskey; early, thick, salmon-fleshed sort; a fine shipper

Perfection or Princess; dark green, netted skin; thick, rich orange-color flesh .............

Rocky Ford; an improved Netted Gem; oblong in shape; flesh green and sweet

Surprise; round, heavily netted; flesh thick, deep salmon-pink .

Tip Top; one of the latest and best; very sweet, deep, salmon-pink flesh ..................

\section{MELON, WATER.}

Black Boulder; very large .............
Cole's Early; medium size, nearly round; dark red flesh; very hardy

Cuban Queen; extra large .........................

Dark Icing; early round; thin skin ............

Dixie; a long variety; good shipper

Duke Jones; early, large, round . . . .

Florida Favorite.

Georgia Rattlesnake or Gypsy

Hungarian Honey; an excellent early variety; perfect globe shape

Ice Cream or Peerless (white seed) $\ldots \ldots \ldots \ldots . . .6$

Jordan's Gray Nonarch ...........................

Kolb's Gem; very popular $\ldots \ldots$

Nammoth Ironclad; good shipper ...............

Mountain Sweet; standard old variety for Northern gardens _.

Phinney's Early; extra early; grown extensively in the North

Pride of Georgia

Seminole; large, oblong....

Sweet-Heart; large globe-shaped: deliciously sweet

The Boss; oblong; dark greeu rind . . . . . . . . . . .

Triumph; a good shipper

Citron, Colorado Preserving .......

Citron, Red-Seeded Preserving

\section{MUSTARD.}

Giant Southern Curled

New Chinese; large leaves for salad . . . . . White London; used for salads and as spinach....

\section{NASTURTIUM.}

Tall Mixed.

$40 \quad 15$

$45 \quad 15$

OKRA OR GUMBO.

Early Dwarf White; extra long white pods, very thick and fleshy; very prolitic............ Improved Dwarf Green Prolific Perkins Mammoth Long Green Podded; good for White Velvet; long tender, velvety white pods....

\section{ONION.}

Proboscidea; seed pods used

\section{WHITE VARIETIES.}

Excelsior Pickling -

Extra Early Pearl; the earliest large white....... 1750000

Portugal or Silver Skin; mild flavored and sown for sets

Southport White Globe; very large, mild flavored_. 225
$50 \quad 15$

$65 \quad 20$ 
Australian Brown; early globe-shaped, distinct in color and a good keeper . _................ Iammoth Prizetaker; ver'y large globe-shaped; productive and mild flavored................. Rhode Island Yellow Cracker; the earliest flat,
yellow onion

Southport Yellow Globe; a good keeper .......... .

Yellow Danvers; handsome, early, round variety; mild flavored and a good keeper.... . ......

Yellow Globe Danvers; the most productive onion

Yellow Dutch or Strasburg; excellent for sets; a good keeper.

\section{RED VARIETIES.}

Extra Early Red Flat; very early and productive.

Large Red Wethersfield; very productive and a good keeper $\ldots \ldots$

Southport Large Red Globe; large, late sort.....

\section{FOREIGN VARIETIES.}

Giant Rocca: very mild, large red globe........
Giant White Italian Tripoli; large flat, quick grow ing, mild flavored variety . . . . . . .

Giant Red Italian Tripoli; similar to the white, excepting in color ............... flavor . . . . . . .

Red Bermuda; very early, flat, pale red...........

White Bermuda; similar to the red except in color

White Adriatic Barletta; small and early; excellent for pickling ..............

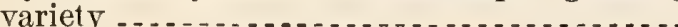

\section{PARSLEY}

Champion Moss Curled

Hamburg or Turnip-Rooted; grown for its edible roots. which resemble small parsnips

Plain or Single; plain dark green leaves .........

\section{PARSNIP.}

Large Hollow Crown or Sugar ...................

Long Smooth White...............................

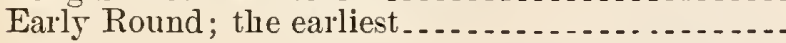

\section{PEAS.}

\section{EXTRA EARLY DWARE VARIETIES.}

* Denotes Trinkled Sorts.

*American ITonder

*Extra Early Premium Gem ... . . . .

IIcLean's Blue Peter

*MicLean's Little Gem.

*Nott's Excelsior. ...

57500

*Tilliam Hurst (new)

\section{EXTRA: EARLY TALL VARIETIES.}

Alaska

First and Best.........

$\because$ Gradus or Prosperity

*Gregory's Surprise or Eclipse ................ 425120

Improved Daniel O'Rourke . . . .

*Laxton's Alpha............................ 400115

New Prolific Early IIarket...................... 4 4 25 1 20

Philadelphia Extra Early..................... 32590

*Thomas Laxton........................ $775 \quad 220$

MEDIUM AND LATE VARIETIES.

*Bliss' Abundance.....

*Bliss' Everbearing.

*Champion of England ........ 3 50 100

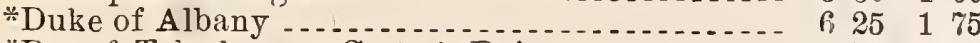

*Dwarf Telephone or Carter's Daisy............. 7775220

*Heroine ......-4 425120

*Horsford's Market Garden................ 350 1. 00

*Improved Stratagem ....................... 725200

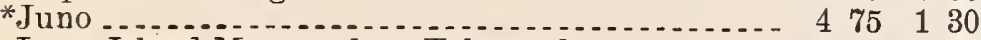

Long Island Mammoth or Telegraph

*McLean's Advancer........................ 3758

Pride of the Market

*Shropshire Hero ........................... 425120

*Telephone, Improved $\ldots \ldots \ldots \ldots$

*Yorkshire Hero . . . _........

Black-Eyed Marrowfat -..................... 22565

White Narrowfat ............................. 22565

Dwarf Grey-seeded Sugar (Edible Pods) ......... 4004115

Mammoth Melting Sugar (Edible Pods) ........... $6 \begin{array}{lllll}6 & 25 & 1 & 75\end{array}$
Celestial; creamy yellow changing to a bright scarlet

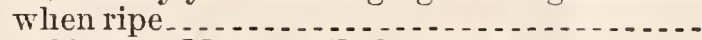

Chinese Giant or Magnum Dulce; the largest sweet

pepper grown
Coral Gem Bouquet; bushes are covered with bright red fruit and have the appearance of a bouquet of corals

Early Dwarf Red Squash . . . .

Golden Dawn; mild and swest

Large Bell or Bull Nose; old standard sort ....... .

Long Red Cayenne (True); very hot and pungent..

Mammoth Golden Queen; large sweet yellow .....

Red Cherry: small fruit, largely used for pickles; very hot ..................................

Red Chili; small bright red, cone-shaped fruit; very hot _.

Red Cluster; small, slender, hot, coral-red fruit...

Ruby King; large, sweet and mild ............
Sweet Mountain or Mammoth; large and mild....

Sweet Spanish Monstrous; large mild flavored

Tabasco; the hottest, small, bright red pepper grown

\section{PUMPKIN.}

Connecticut Field; the old common yellow, field variety -..............................

Cushaw or Crookneck; very large, cream color flesh of fine quality.

Japanese Pie; thick, fine grained, sweet flesh; seeds are curiously marked

Jonathan; large, crookneck, bottle-shaped; a good keeper

Large Cheese or Kentucky Field

Large Tours or Mammoth; very large, mostly used for cattle feeding

Livingston's Cushaw; Green aud white striped, fine grained yellow flesh

Nammoth Bright Red Etampes; very large ..... .

Quaker Pie; shape, oval tapering toward each end Sugar or Rhode Island Sweet; the popular pie pumpkin of New England

Tennessee Sweet Potato; pear shaped with white, sweet, thick flesh.

Winter Luxury; round, yellow, finely netted; rery productive and a good keeper.

\section{RADISH.}

\section{TURNIP-SHAPED VARIETIES.}

Early Deep Scarlet White-Tipped Forcing; ready for use in about 20 days

Early Deep Scarlet Forcing; crisp and tender..... Early Searlet White-Tipped

Early Round Dark Red or Scarlet Button; an excellent forcing variety. -

Extra Early Scarlet Globe; very handsome, crisp and tender.

Non Plus Ultra or Fireball; one of the best, earliest red foreing varieties

Philadelphia White Box; always crisp and tender.

Rosy Gem; fine for forcing ..................

\section{OLIVE.SHA PED VARIETIES.}

French Breakfast; pink tipped with white........ Golden Yellow; fine for forcing .....

Scarlet Olive-Shaped; very early .....

White Olive-Shaped; early, crisp and mild

Vick's Scarlet Globe.

\section{LONG VARIE'TIES.}

Brightest Scarlet, White-Tipped; very distinct, flesh crisp and sweet. ......................

Chartier or Shepard Half-Long Deep Scarlet or Paris Beauty.......... Long Scarlet Shnrt Top . . . . . . . . . . . . . . . . . . . Wood's Early Frame; excellent for forcing......

$\begin{array}{lll}45 & 15 & 5 \\ 40 & 15 & 5 \\ 40 & 15 & 5 \\ & & \\ 35 & 12 & 5 \\ 40 & 15 & 5 \\ & & \\ 45 & 15 & 5 \\ & & \\ 45 & 15 & 5 \\ 40 & 15 & 5 \\ 35 & 12 & 5\end{array}$

\section{SUMMIRE VARIETIES.}

Giant White Stuttgart; large globe shaped, withstands heat .....................................

$\begin{array}{lll}35 & 12 & 5 \\ 50 & 15 & 5 \\ 50 & 15 & 5 \\ 40 & 15 & 5 \\ 40 & 15 & 5 \\ 45 & 15 & 5\end{array}$

$\begin{array}{lll}35 & 12 & 5 \\ 35 & 12 & 5 \\ 35 & 12 & 5 \\ 40 & 15 & 5 \\ 30 & 12 & 5 \\ 35 & 12 & 5\end{array}$


Golden Summer; flesh solid and crisp

Large White Summer Turnip

Long White Naples; very mild

Long White Strasburg, flesh is always b. tender

California Mammoth White; a good keeper ....... . Celestial or White Chinese; good for either winter or summer.

Half-Iong Black Spanish; a new variety ........

Long Black Spanish; bardy late variety .........

Long White Spanish; milder flavored than Black Spanish

Round Black Spanish; globe-shaped.............

Rose China Winter

RHUBARB OR PIE PLANT.

Linnæus; early, very large leaves ............... 100

Victoria; later than the above................. 100

\section{SALSIFY OR OYSTER PLANT.}

Tong White French

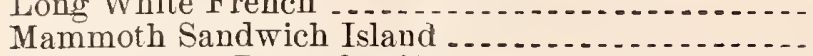

Scorzonera or Black Salsify.

\section{SPINACF.}

Bloomsdale Savoy Leaved; the hardiest and most popular sort

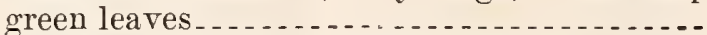

Long Standing; dark green, stands longer than any other variety before running to seed .........

Monstrous Viroflay; useful for either spring or fall sowing, very productive.....................

Prickly or Winter; very hardy

Round Leaved; an old standard variety ...........

Victoria; thick, dark green leaves, good for spring sowing -

New Zealand; a distinct variety, growing well in

\section{SQUASH.}

Boston Narrow; bright orange; oval shape.

Delicata; small; rich and dry

Early White Bush Scalloped or Patty Pan .......

Early Yellow Bush Scalloped . .

Mammoth White Bush Scalloped.

Giant Summer Crookneck; very large

Golden Summer Crookneck; early

Golden Custard Bush; very large, golden yellow..

Essex Hybrid; a good keeper.

Extra Early Orange Marrow

Faxon; fine winter variety

Fordhook; a good all-the-year-round squash; very weet and dry.

Hubbard Improved; the best popular winter squash

Hubbard Chicago Warted; very large; rich, dark green with bright, orange-red skin .

Mammoth Chili; the largest of all, sometimes weighing $200 \mathrm{lus}$

Pike's Peak or Sibley; very productive; dry and sweet

Vegetable Marrow; white-fleshed variety for summer and fall use

Winter Crookneck; well-known old favorite

$\begin{array}{ccr}\text { Lb. } & \text { 3/4 Lb. } & \text { O\%. } \\ 35 & 12 & 5 \\ 40 & 15 & 5 \\ 40 & 15 & 5\end{array}$

$35 \quad 12$

$40 \quad 15$

$45 \quad 15$

$65 \quad 20$

$40 \quad 15$

$35 \quad 12$

$35 \quad 12$

$35 \quad 12$

Connecticut Seed Leaf
Havana (True)

TOMATO.

Acme: early, round, smooth of a peculiar pink

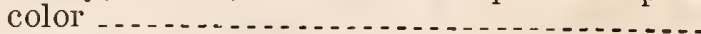

Atlantic Prize; very early; smooth and solid.....

Crimson Cushion; large and solid.

Dwarf Aristocrat; a new upright bush; fine for

Dwarf Champior; compact and upright growth early, smooth purplish-red fruit ............

Earliana, Spark's; the earliest, large, smooth red;

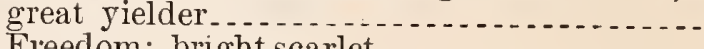

Early Freedom; bright scarlet.

Golden Queen; large, smooth .................

Ignotum; early, large deep red.

Livingston's Beauty; large, smooth pinkish red...

$\begin{array}{ll}30 & 10 \\ 30 & 10\end{array}$

Livingston's Magnus; a large purplish-red variety; early and productive

Livinoston's Paragon; very productive

Livingston's Perfection; early, blood red, having few seeds; very productive

$\begin{array}{rrr}70 & 22 & 6 \\ 90 & 30 & 10\end{array}$

$150 \quad 50 \quad 15$

10 Lbs. Lb. $1 / 4 \mathrm{Lb}$.

$130 \quad 15 \quad 6$

$\begin{array}{llll}130 & 15 & 6\end{array}$

$120 \quad 14$

$130 \quad 15$

$160 \quad 20$

$120 \quad 14$

$130 \quad 15$

$50 \quad 15$

$\begin{array}{lcr}\text { Lb. } & 1 / 4 \mathrm{Lb}, & \text { Oz. } \\ \mathbf{5 0} & 15 & 5 \\ 60 & 18 & 6 \\ 50 & 15 & 5 \\ 50 & 15 & 5 \\ 55 & 18 & 5 \\ 65 & 20 & 6 \\ 55 & 18 & 5 \\ 65 & 20 & 6 \\ 55 & 18 & 5 \\ 65 & 20 & 6 \\ 75 & 25 & 7\end{array}$

Mikado or Turner's Hybrid; large, purplish-red, potato-leaved variety, containing few seeds..

New Stone; large, round, deep-red fruit which is very heavy; very productive.

Pear-Shaped Red; used chiefly for preserving.....

Pear-Shaped Yellow . . . . . . . . . . . . . . . . . . . . . .

Ponderosa; the largest fruited sort grown .........

Red Cherry; very small, round and smooth; good

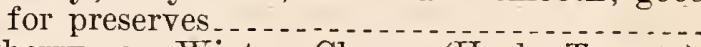

Strawberry or Winter Cherry (Husk Tomato); small, yellow fruit enclosed in a husk; fine

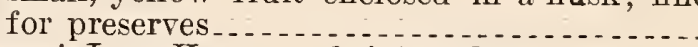

Thorburn's Long Keeper: a bright red. early variety noted for its long keeping qualities ........

Trophy; the old standard late variety; very solid and large, bright red ..........................

\section{TURNIP.}

Amber Globe.... with purplish red top

Early Snowball; early, round, white variety .....

Early White Flat Dutch: tlat, white and very sweet

Extra Early Purple-Top Milan; the earliest variety

Golden Ball or Orange Jelly; very early, small Whirety Globe, Strap-Leaved; early ............

Large White Globe, Strap-Leaved; early
Red-Top Strap-Leaved; the well known old standard variety

Red-Top White Globe ........

Seven Top; used in the South for greens.......

White Egg; good for either early or late sowing...

75257 White Milan; extra early white variety .........

60186 Yellow Aberdeen

Yellow Stone; sh yellow and sweet

RUTA HAGA VARIETIES.

$7525 \quad 7$ Budlong White Rock; the earliest American variety

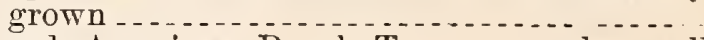

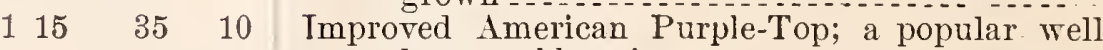
known old variety-.........

$9030 \quad 10$ Large White French; flesh white, sweet and very

$7525 \quad 7 \quad$ Long Island Purple-Top; the best..................

$50 \quad 15 \quad 5 \quad$ White Sweet German; fine winter variety $\ldots . . . . .$.

$160 \quad 45 \quad 15$

$\begin{array}{llll}1 & 60 & 45 & 15\end{array}$

$\begin{array}{lllll}4 & 50 & 130 & 35\end{array}$

$190 \quad 55 \quad 15$

$175 \quad 50 \quad 15$

$\begin{array}{lll}250 & 75 & 20\end{array}$

$\begin{array}{lll}250 & 75 & 20\end{array}$

$\begin{array}{llll}175 & 50 & 15\end{array}$

$\begin{array}{llll}175 & 50 & 15\end{array}$

$\begin{array}{lll}160 & 45 & 15\end{array}$

$\begin{array}{llll}160 & 45 & 15\end{array}$

$175 \quad 50 \quad 15$

$\begin{array}{llll}160 & 45 & 15\end{array}$

$\begin{array}{llll}160 & 45 & 15\end{array}$

$200 \quad 60 \quad 18$

$\begin{array}{llll}160 & 45 & 15\end{array}$

$\begin{array}{llll}1 & 75 & 50 & 15\end{array}$

$\begin{array}{llll}175 & 50 & 15\end{array}$

$\begin{array}{lll}325 & 90 & 25\end{array}$

$\begin{array}{llll}1 & 75 & 50 & 15\end{array}$

$200 \quad 60 \quad 18$

$175 \quad 50 \quad 15$

$\begin{array}{llll}175 & 50 & 15\end{array}$

30

30

40

40

30

30

$25 \quad 10$

$35 \quad 12$

30 10

$35 \quad 12$

$65 \quad 20$

$30 \quad 10$

\section{HERBS (Seeds and Plants).}

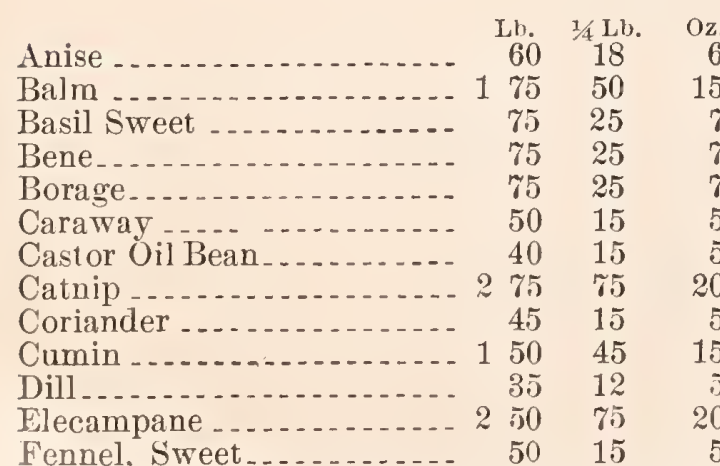

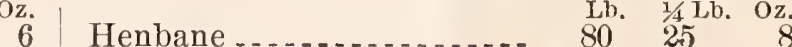

Horehound

Hop-seed, pkt. $5 \mathrm{c}$............

泟

Lavender............... $150 \quad 45 \quad 15$

Lovage (Levisticum) _...... 1 75 $50 \quad 50 \quad 15$

Narjoram, Pot_._._._._. $20060 \quad 18$

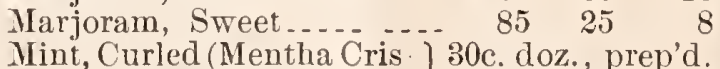

pa), Strong Plants . . . . $\$ 1$ per 100

$\left.\begin{array}{r}\text { Mint. Peppermint (Mentha } \\ \text { Piperita), Strong Plants }\end{array}\right\} \begin{array}{r}\$ 6 \text { per } 1.000 \text { by } \\ \text { exp. not prep d }\end{array}$
Rosemary .............. 200

Saffron

Sage, Imp. Broad-leaved... 125

Savory, Summer.......... 60

Savory, Winter......... 150

Sorrel, Broad-Leaved ...... 80

Tansy ...............2 50

Tarragon . . . . . .

Thyme

Wormwood _......... 150
1/4 Lb. Oz

60

25
18

18

45

$\therefore$

75 


\section{CLOVENA NURSERIES}

\section{Discount Sheet to the Grade}

\section{ON RICHMOND SEEDS}

New York, January, 1906.

To Seed Dealers :

We allow following special discounts from our 1906 Seed Price List, sent herewith, to dealers and the trade:

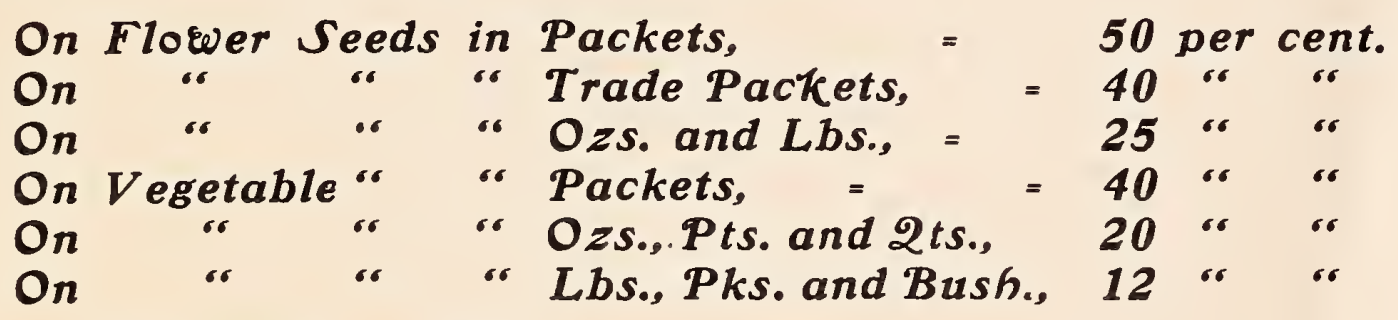

We should be pleased to figure on your wants.

New York Market Gardeners' Ass'n

P. O. Box 1423

NEW YORK, N. Y. 


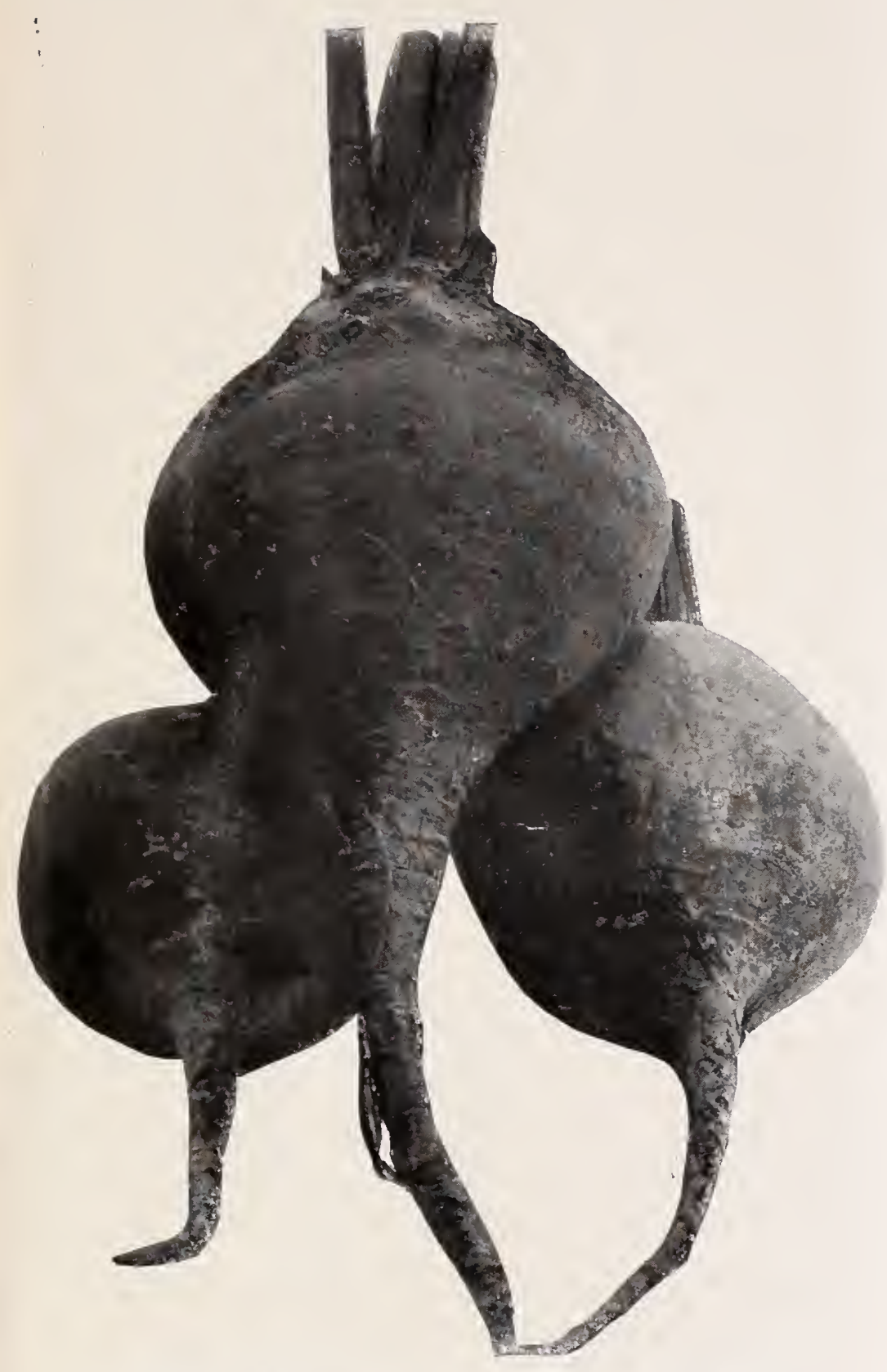

BEET, IMPROVED EARLY ECLIPSE.

Lb., 40c.; 1/4 lb., 15c.; oz., 5c.

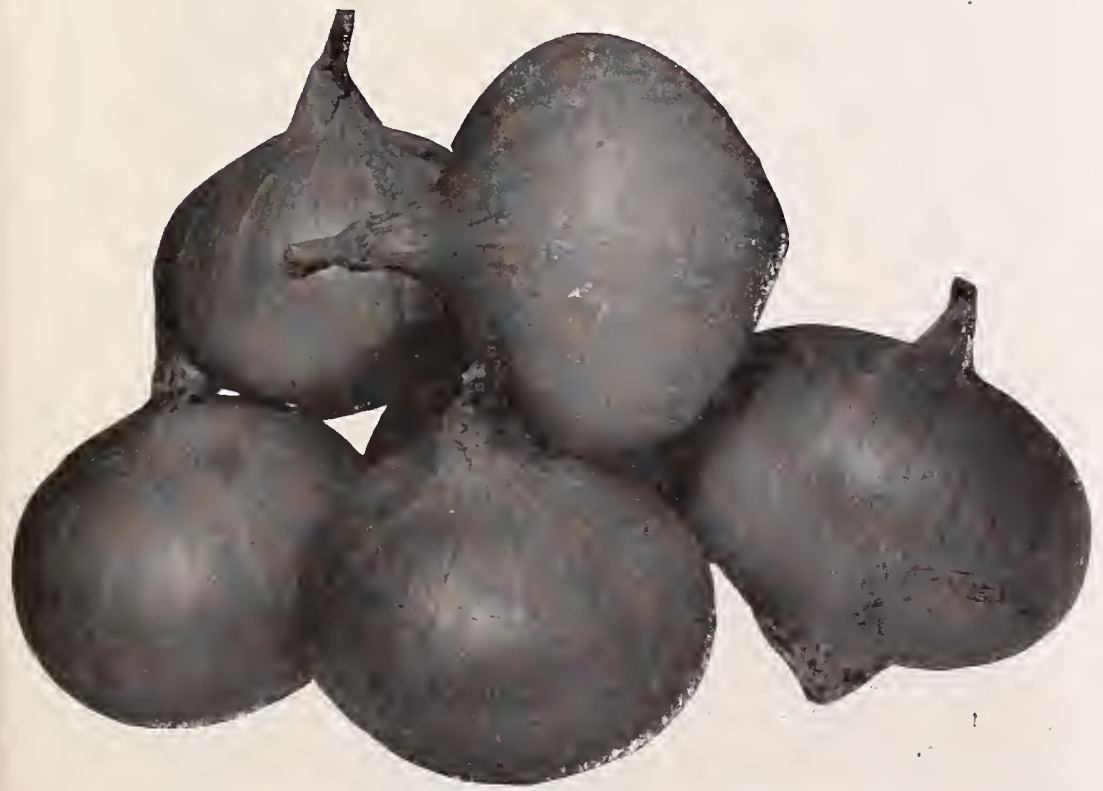

ONION, AUSTRALIAN BROWN.

Early Maturing and the best Keeper.

Lb., $\$ 1.00$; 1/4 lb., 30c.; oz., 10c.

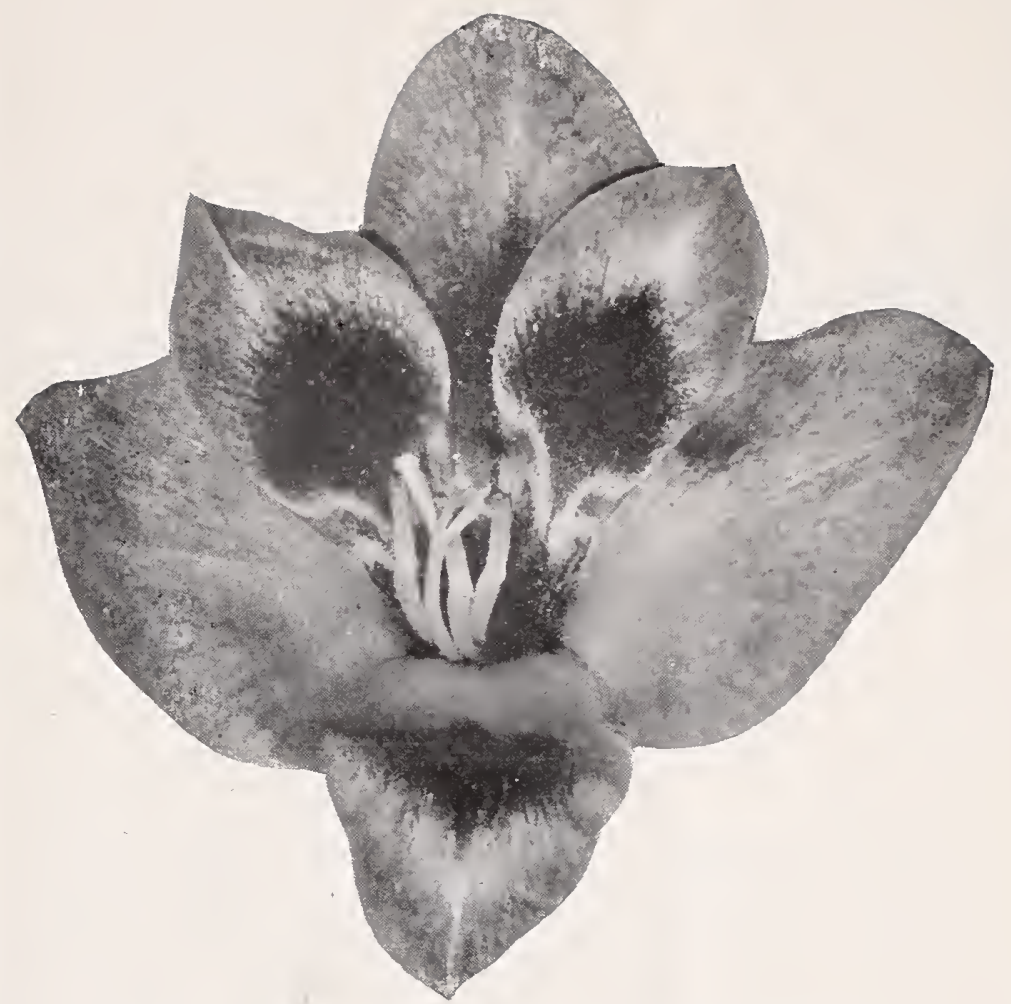

GLA DIOLI CLOVENA HYBRIDS.

New Creations in Form and Color. Finest Strains in Cultivation.

Doz. $\quad 100$

1,000

No, 1 Bulbs, 1 inch and up to $1 \frac{3}{4}$ inch in diameter $\$ 022 \$ 155$

$\$ 1400$

“ 2 Bulbs, $7 / 8$ inch to 1 inch in diameter, strong blooming size...............

1000

3 Bulbs, $3 / 4$ inch to $\%$ inch in diameter, fine blooming size .................

“ 4 Bulbs, $5 \%$ inch to $3 / 4$ inch in diameter, most will bloom................. 12

“ 5 Bulbs, $1 / 2$ inch to $5 / 3$ inch in diameter, many will bloom ................

“ 6 Bulbs, $3 / 8$ inch to $1 / 2$ inch in diameter, many will bloom ................

" 7 Bulbs, $1 / 4$ inch to $3 / 8$ inch in diameter

" 8 Bulblets

“ 1 May, true flesh color, flaked and striped daybreak pink..............

$15 \quad .95 \quad 800$

$12 \quad 75 \quad 600$

$10 \quad 60$

$10 \quad 40 \quad 300$

-- 30

Begonia Grandiflora, single mixed colors _ Begonia Grandiflora, double mixed colors _ Caladium Esculentum (Elephants Ears):

6 to 8 inch, fine for potting ..........

7 to 9 “ extra value.......... 30

8 to 10 " very fine.............. 45

9 to 11 " extra large ............

Dahlias, double show flowering, mixed ...

Dahlias, single seedlings, mixed .........

Iris, Japan, Hybrid mixed..............

Tuberose, Dwarf Pearl Mammoth. . . . . . .

Tuberose, Dwarf Pearl, first size, 4 inch. -

Tuberose, Dwarf Pearl, No. 2 size, 3 inch to 4 inch
400

135

175

275

325

400

350

400

100

65

3750

1500

1600

2400

3000

3500

3000

3500

700 


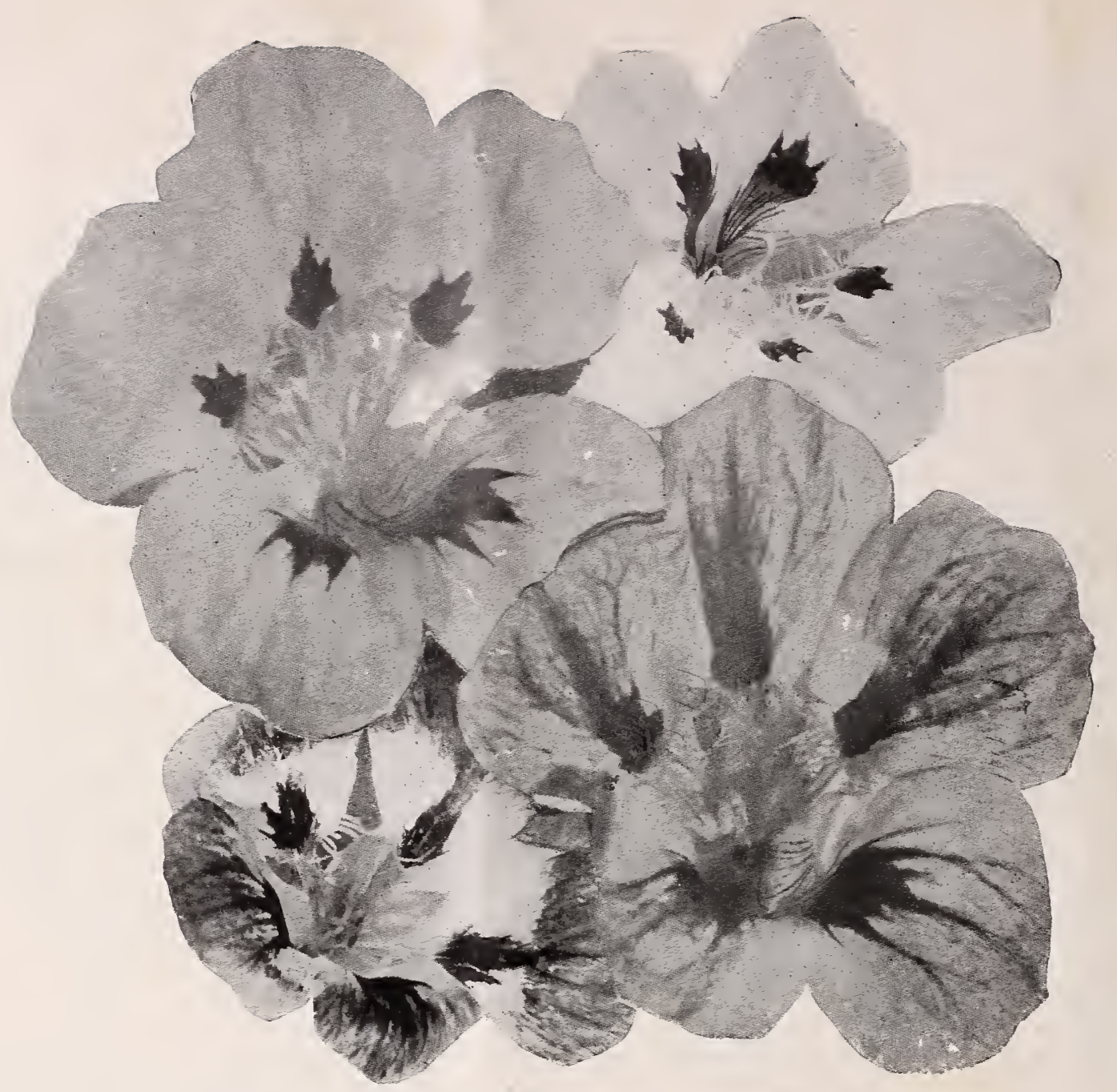

2484-NASTUR'TIUM DWARF OR TOM THUMB.

Our Own Mixture, Jb., 60c.; $\frac{1}{4}$ lb., 20c.; oz., 10c.

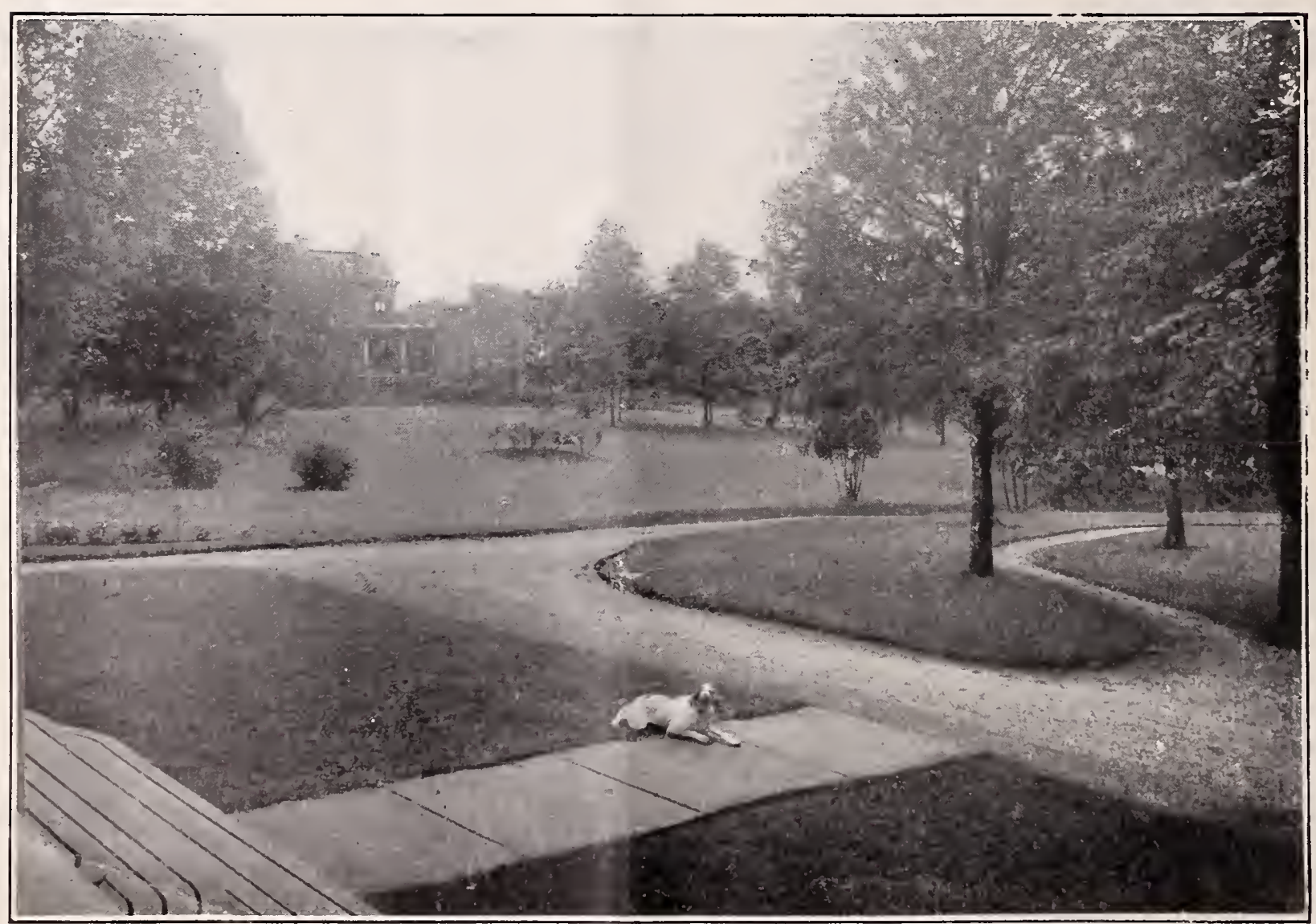

LAWN GRASS SEED, CLOVENA MIXTURE.

Extra heavy, clean, tested seed, weighs $20 \mathrm{lbs}$. to the bushel, and 80 to $85 \%$ grows. (Jomposed of the finest grasses for making lawns, putting greens, tennis courts, cricket grounds, renovating spots in old lawns, etc. Superior for exposed situations, producing, under favorable conditions, permanent, elastic and resistant velvet turf of emerald green in six to eight weeks. For first-class work, sow four bushels ( $80 \mathrm{lbs}$.) to the acre; and good results can be obtained with three bushels. Price, quart, 15 cents; peck, $\$ 1.00$; bushel, $\$ 3.00$. 